\title{
Smoothing Properties and Retarded Estimates for Some Dispersive Evolution Equations
}

\author{
J. Ginibre ${ }^{1}$ and G. Velo ${ }^{2}$ \\ 1 Laboratoire de Physique Théorique et Hautes Energies*, Université de Paris XI, Bâtiment 211, \\ F-91405 Orsay, France \\ 2 Dipartimento di Fisica, Università di Bologna and INFN, Sezione di Bologna, Italy
}

Received June 18, 1991

\begin{abstract}
Smoothing properties, in the form of space-time integrability properties, play an important role in the study of dispersive evolution equations. A number of them follow from a combination of general arguments and specific estimates. We present a general formulation which makes the separation between the two types of ingredients as clear as possible, and we illustrate it with the examples of the Schrödinger equation, of the wave equation, and of a class of $1+1$ dimensional equations related to the Benjamin-Ono equation. Of special interest for the Cauchy problem are retarded estimates expressed in terms of those properties. We derive a number of such estimates associated with the last example, and we mention briefly an application of those estimates to the Cauchy problem for the generalized Benjamin-Ono equation.
\end{abstract}

\section{Introduction}

A large amount of work has been devoted in the last twenty years to the spacetime integrability properties of solutions and to the smoothing properties of dispersive partial differential equations of the type

$$
\partial_{t} u-L u=f,
$$

where $u$ is the unknown (possibly vector valued) function, defined in space-time $\mathbb{R}^{n+1}, L$ is a skew adjoint operator in some Hilbert space $\mathscr{H}$ of functions of the space variable, for instance $\mathscr{H}=L^{2}\left(\mathbb{R}^{n}\right)$, and $f$ can be a given external source term, or a (possibly non-linear) function of the unknown function $u$, or a combination of both [3-10, 12-24]. Let $U(t)=\exp (t L)$ be the unitary one parameter group in $\mathscr{H}$ generated by $L$. The Cauchy problem for (1.1) with initial data $u(t=0)=u_{0}$ is formally equivalent to the integral equation

$$
u(t)=U(t) u_{0}+\int_{0}^{t} d \tau U(t-\tau) f(\tau)
$$

\footnotetext{
* Laboratoire associé au Centre National de la Recherche Scientifique
} 
and an essential role in the treatment of that problem is played by the properties of the operators

$$
\begin{gathered}
u_{0} \rightarrow U(t) u_{0}, \\
f \rightarrow \int_{0}^{t} d \tau U(t-\tau) f(\tau)
\end{gathered}
$$

A number of those properties are expressed in terms of norms involving space and time integrals of $f$ and of the images of $u_{0}$ and $f$, and possibly space derivatives of those functions. They can be regarded as smoothing properties of the equation in so far as less derivatives occur on $u_{0}$ and $f$ than on their images.

The formulation and proof of those properties combine abstract arguments and specific estimates, which are not always clearly separated in the existing applications. In the present paper, we first present a general framework which makes that separation as clear as possible, and illustrate it with several examples taken from the literature, namely the Schrödinger equation $[7,14,24]$, the wave equation $[3,19]$ and a class of equations in $1+1$ dimensional space time which is related to the generalized Benjamin-Ono equation $[15,17]$. That material is presented in Sect. 2.

The previous framework allows for a satisfactory treatment of the term $U(\cdot) u_{0}$ in (1.2), but it may not be sufficient to treat the integral term in (1.2) because of the retardation property $\tau \leq t$ in the time integration. We consider that problem in Sect. 3. We first identify two cases, already widely used in the applications, where the retardation introduces no additional complication. We then come back to the $1+1$ dimensional class of examples mentioned above, and study in some detail the cases where the retardation has a non-trivial effect. We prove in particular that under suitable additional assumptions, the retardation essentially does not spoil the previous space-time integrability properties. The results are contained in Propositions 3.2-3.6. We finally mention briefly an application of the previous results to the Cauchy problem for the generalized Benjamin-Ono equation.

Technical proofs are collected in an Appendix.

We conclude this introduction by giving some notation that will be used freely in this paper. We denote by $\|\cdot\|_{r}$ the norm in $L^{r} \equiv L^{r}\left(\mathbb{R}^{n}\right)$. Pairs of conjugate indices are written as $r$ and $\bar{r}$, where $2 \leq r \leq \infty$ and $r^{-1}+\bar{r}^{-1}=1$. With any $r, 1 \leq r \leq \infty$, we associate the variable $\alpha(r)=1 / 2-1 / r$. For any interval $I$ of $\mathbb{R}$, possibly $\mathbb{R}$ itself, and for any $q, r, 1 \leq q, r \leq \infty$, we denote by $L^{q}\left(I, L^{r}\right)$ the space of measurable functions $f$ from $I$ to $L^{r}$ such that $\|f\|_{r} \in L^{q}(I)$. In order to avoid possible sources of confusion, especially in $1+1$ space-time dimension, we shall indicate by subscripts $t$ and $x$ the name of the variables in those spaces. For instance $L_{t}^{q}\left(\mathbb{R}, L_{x}^{r}\right)$ (respectively $L_{x}^{q}\left(\mathbb{R}, L_{t}^{r}\right)$ if $n=1$ ) denotes the space of $L^{q}$ functions of the time variable (respectively of the space variable) with values in the $L^{r}$ space of the space variable (respectively of the time variable). We denote by $\mathscr{S}_{n+1}$ and $\mathscr{S}_{n+1}^{\prime}$ the spaces of rapidly decreasing functions and of tempered distributions in $\mathbb{R}^{n+1}$, by $\mathscr{F}$ and $\mathscr{F}{ }_{n}$ the Fourier transform in $\mathbb{R}^{n+1}$ and in $\mathbb{R}^{n}$ (of the space variable) respectively. Finally, we denote by $*,{ }_{x}$ and $*_{t}$ the convolution in space-time, in space and in time respectively.

A preliminary version of the contents of this paper has been presented in [6]. 


\section{General Framework and Examples}

The space time integrability properties associated with the equation (1.1) can be considered from two different points of view, namely the operator point of view [7] and the point of view of the Fourier transform [22]. We begin with an abstract result which is relevant to both and which although elementary, lies at the root of the matter. For any vector space $\mathscr{D}$, we denote by $\mathscr{D}_{a}^{*}$ its algebraic dual, by $\mathscr{L}_{a}(\mathscr{D}, X)$ the space of linear maps from $\mathscr{D}$ to some other vector space $X$, and by $\langle\varphi, f\rangle_{\mathscr{D}}$ the pairing between $\mathscr{D}_{a}^{*}$ and $\mathscr{D}$ (with $f \in \mathscr{D}$ and $\varphi \in \mathscr{D}_{a}^{*}$ ), which we take to be linear in $f$ and anti linear in $\varphi$.

Lemma 2.1. Let $\mathscr{H}$ be a Hilbert space, $X$ a Banach space, $X^{*}$ the dual of $X$, and $\mathscr{D}$ a vector space densely contained in $X$. Let $T \in \mathscr{L}_{a}(\mathscr{D}, \mathscr{H})$ and let $T^{*} \in$ $\mathscr{L}_{a}\left(\mathscr{H}, \mathscr{D}_{a}^{*}\right)$ be its adjoint, defined by

$$
\left\langle T^{*} v, f\right\rangle_{\mathscr{D}}=\langle v, T f\rangle, \quad \forall f \in \mathscr{D}, \quad \forall v \in \mathscr{H},
$$

where $\langle$,$\rangle is the scalar produit in \mathscr{H}$ (antilinear in the first argument). Then the following three conditions are equivalent.

(1) There exists $a, 0 \leq a<\infty$ such that for all $f \in \mathscr{D}$,

$$
\|T f\| \leq a\|f ; X\| \text {. }
$$

(2) $\mathscr{R}\left(T^{*}\right) \subset X^{*}$, and there exists $a, 0 \leq a<\infty$, such that for all $v \in \mathscr{H}$

$$
\left\|T^{*} v ; X^{*}\right\| \leq a\|v\| .
$$

(3) $\mathscr{R}\left(T^{*} T\right) \subset X^{*}$ and there exists $a, 0 \leq a<\infty$, such that for all $f \in \mathscr{D}$,

$$
\left\|T^{*} T f ; X^{*}\right\| \leq a^{2}\|f ; X\|,
$$

where $\|\cdot\|$ denotes the norm in $\mathscr{H}$. The constant a is the same in all three parts. If one of (all) those conditions is (are) satisfied, the operators $T$ and $T^{*} T$ extend by continuity to bounded operators from $X$ to $\mathscr{H}$ and from $X$ to $X^{*}$ respectively.

Proof. From the fact that $\mathscr{D}$ is densely contained in $X$, it follows that $X^{*}$ is a subspace of $\mathscr{D}_{a}^{*}$.

(1) $\Rightarrow$ (2). Let $v \in \mathscr{H}$. Then, for all $f \in \mathscr{D}$

$$
\left|\left\langle T^{*} v, f\right\rangle_{\mathscr{D}}\right|=|\langle v, T f\rangle| \leq\|v\|\|T f\| \leq a\|v\|\|f ; X\| .
$$

(2) $\Rightarrow(1)$. Let $f \in \mathscr{D}$. Then for all $v \in \mathscr{H}$

$$
|\langle v, T f\rangle|=\left|\left\langle T^{*} v, f\right\rangle_{\mathscr{D}}\right| \leq\left\|T^{*} v ; X^{*}\right\|\|f ; X\| \leq a\|v\|\|f ; X\| .
$$

Clearly (1) and (2) imply (3), and therefore (1) or (2) imply (3).

(3) $\Rightarrow(1)$. Let $f \in \mathscr{D}$. Then

$$
\langle T f, T f\rangle=\left\langle T^{*} T f, f\right\rangle_{\mathscr{D}} \leq\left\|T^{*} T f ; X^{*}\right\|\|f ; X\| \leq a^{2}\|f ; X\|^{2} .
$$

The following corollary is extremely useful.

Corollary 2.1. Let $\mathscr{H}, \mathscr{D}$ and two triplets $\left(X_{i}, T_{i}, a_{i}\right), i=1,2$, satisfy the conditions of Lemma 2.1. Then for all choices of $i, j=1,2, \mathscr{R}\left(T_{i}^{*} T_{j}\right) \subset X_{i}^{*}$, and for all $f \in \mathscr{D}$,

$$
\left\|T_{i}^{*} T_{j} f ; X_{i}^{*}\right\| \leq a_{i} a_{j}\left\|f ; X_{j}\right\| .
$$


In particular $T_{i}^{*} T_{j}$ extends by continuity to a bounded operator from $X_{j}$ to $X_{i}^{*}$, and (2.4) holds for all $f \in X_{j}$.

We now describe the operator point of view, which provides the basic example for the situation of Lemma 2.1. Let $\mathscr{H}$ be a Hilbert space, and $U$ a unitary strongly continuous one parameter group in $\mathscr{H}$. Let $I$ be an interval of $\mathbb{R}$, possibly $\mathbb{R}$ itself. We define the bounded operator $A$ from $L^{1}(I, \mathscr{H})$ to $\mathscr{H}$ by

$$
A f=\int_{I} d \tau U(-\tau) f(\tau) .
$$

Then its adjoint $A^{*}$ is the operator

$$
A^{*} v(t)=U(t) v
$$

from $\mathscr{H}$ to $L^{\infty}(I, \mathscr{H})$, where duality is defined by the scalar products in $\mathscr{H}$ and in $L^{2}(I, \mathscr{H})$, so that $A^{*} A$ is the bounded operator from $L^{1}(I, \mathscr{H})$ to $L^{\infty}(I, \mathscr{H})$ given by

$$
A^{*} A f=\int_{I} d \tau U(t-\tau) f(\tau)
$$

which we shall write more concisely as $A^{*} A f=U *_{t} f$. Clearly the conditions of Lemma 2.1 are satisfied with $X=L^{1}(I, \mathscr{H}), T=A, a=1$, and $\mathscr{D}$ any dense subspace of $X$. The relevance of the operator point of view for the Cauchy problem for the equation (1.1) is obvious from a comparison of (1.2) with (2.6), (2.7).

The operator point of view, Lemma 2.1 and Corollary 2.1 immediately yield the well known properties of the Schrödinger equation in $\mathbb{R}^{n}$,

$$
i \partial_{t} u=-(1 / 2) \Delta u
$$

where $\Delta$ is the Laplace operator. The associated unitary group $U$ in $\mathscr{H}=L_{x}^{2}$ is given by

$$
U(t)=\mathscr{F}_{n}^{-1} \exp \left(-i(t / 2) \xi^{2}\right) \mathscr{F}_{n},
$$

or equivalently

$$
U(t)=(2 \pi i t)^{-n / 2} \exp \left(i x^{2} / 2 t\right) *_{x} .
$$

It follows from (2.8) that $U(t)$ extends to a bounded operator from $L_{x}^{1}$ to $L_{x}^{\infty}$ with

$$
\|U(t) v\|_{\infty} \leq(2 \pi|t|)^{-n / 2}\|v\|_{1}
$$

and by interpolation with unitarity in $\mathscr{H}$, to a bounded operator from $L_{x}^{\bar{r}}$ to $L_{x}^{r}$ with

$$
\|U(t) v\|_{r} \leq(2 \pi|t|)^{-n \alpha(r)}\|v\|_{\bar{r}}
$$

for $2 \leq r \leq \infty$, with $\alpha(r)=1 / 2-1 / r$. It follows then from the Hardy-LittlewoodSobolev (HLS) inequality [11, p. 117] that the operator $A^{*} A$ defined by (2.7) with $I=\mathbb{R}$ extends to a bounded operator from $X_{r}=L_{t}^{\bar{q}}\left(\mathbb{R}, L_{x}^{\bar{r}}\right)$ to $X_{r}^{*}=L_{t}^{q}\left(\mathbb{R}, L_{x}^{r}\right)$ for $0 \leq 2 / q=n \alpha(r)<1$. The range of values of $r$ is $2 \leq r \leq \infty$ for $n=1$, $2 \leq r<\infty$ for $n=2$ and $2 \leq r<2^{*} \equiv 2 n /(n-2)$ for $n \geq 3$. In particular, the condition (3) and therefore all conditions of Lemma 2.1 are satisfied for the operators defined by $(2.5)-(2.7)$ with $I=\mathbb{R}$ and the previous spaces $X_{r}$. The implication $(3) \Rightarrow(2)$ yields the estimate

$$
\left\|U(\cdot) v ; L_{t}^{q}\left(\mathbb{R}, L_{x}^{r}\right)\right\| \leq C\|v\|_{2}
$$


for all $v \in L_{x}^{2}$, and Corollary 2.1 yields the estimate

$$
\left\|U *_{t} f ; L_{t}^{q_{1}}\left(\mathbb{R}, L_{x}^{r_{1}}\right)\right\| \leq C\left\|f ; L_{t}^{\bar{q}_{2}}\left(\mathbb{R}, L_{x}^{\bar{r}_{2}}\right)\right\|
$$

for all pairs $(q, r),\left(q_{1}, r_{1}\right)$ and $\left(q_{2}, r_{2}\right)$ as above. In particular, the well known but remarkable decoupling of $r_{1}$ and $r_{2}$ in (2.11) is a special case of the trivial Corollary 2.1 .

We now turn to the point of view of the Fourier transform [22]. We consider an $n$-dimensional piecewise smooth surface $\Sigma$ in $\mathbb{R}^{n+1}$, and a positive measure $\sigma$ supported by $\Sigma$ and such that $\sigma \in \mathscr{S}_{n+1}^{\prime}$, so that $\mathscr{S}_{n+1 \mid \Sigma} \subset L^{r}(\Sigma, \sigma)$ for all $r, 1 \leq r \leq \infty$. We try to implement the situation of Lemma 2.1 by taking $\mathscr{H}_{\sigma}=L^{2}(\Sigma, \sigma), \mathscr{D}=\mathscr{S}_{n+1}$ and by defining the operators $B$ and $B_{\sigma}^{*}$ by

$$
\begin{gathered}
B f=\left.\mathscr{F} f\right|_{\Sigma}, \\
B_{\sigma}^{*} v=\mathscr{F}^{-1}(v \sigma)
\end{gathered}
$$

for all $f \in \mathscr{S}_{n+1}$ and $v \in \mathscr{H}_{\sigma}$. The adjoint $B_{\sigma}^{*}$ is defined by the scalar products in $L^{2}\left(\mathbb{R}^{n+1}\right)$ and in $L^{2}(\Sigma, \sigma)$, and therefore depends on $\sigma$. The operator $B_{\sigma}^{*} B$ is given by

$$
B_{\sigma}^{*} B f=(2 \pi)^{-(n+1) / 2} \mathscr{F}^{-1}(\sigma) * f
$$

for all $f \in \mathscr{S}_{n+1}$. More explicitly, with $z \in \mathbb{R}^{n+1}$ and $\zeta \in \Sigma$,

$$
\begin{gathered}
B f(\zeta)=(2 \pi)^{-(n+1) / 2} \int d z f(z) \exp (-i z \zeta), \\
\left(B_{\sigma}^{*} v\right)(z)=(2 \pi)^{-(n+1) / 2} \int d \sigma(\zeta) v(\zeta) \exp (i z \zeta), \\
\left(B_{\sigma}^{*} B f\right)(z)=(2 \pi)^{-(n+1)} \int d \sigma(\zeta) d z^{\prime} f\left(z^{\prime}\right) \exp \left(i\left(z-z^{\prime}\right) \zeta\right) .
\end{gathered}
$$

Clearly $B$ extends to a bounded operator from $L^{1}\left(\mathbb{R}^{n+1}\right)$ to $\mathscr{C}_{b}(\Sigma)$ and $B_{\sigma}^{*}$ also defines a bounded operator from $L^{1}(\Sigma, \sigma)$ to $\mathscr{C}_{b}\left(\mathbb{R}^{n+1}\right)$.

We are interested in spaces $X$ implementing the situation of Lemma 2.1. For a fixed surface $\Sigma$, we shall call admissible the pairs $(\sigma, X)$ such that the conditions of Lemma 2.1 hold for $\mathscr{H}=\mathscr{H}_{\sigma}, \mathscr{D}=\mathscr{S}_{n+1}$ and $T=B$. In the present case, one can take advantage of the existing topology on $\mathscr{D}$ to replace $\mathscr{D}_{a}^{*}$ by $\mathscr{D}^{*}=\mathscr{S}_{n+1}^{\prime}$. Before turning to examples, we give a general invariance property of the notion of admissible pairs. We study the effect of multiplying $\sigma$ by a weight function $w^{-1}$ defined in $\mathbb{R}^{n+1}$, which we assume to be suitably regular, almost everywhere positive and finite, both in $\mathbb{R}^{n+1}$ and on $\Sigma$, and such that $w^{-1} \sigma \in \mathscr{S}_{n+1}^{\prime}$. Let $(\sigma, X)$ be an admissible pair, let $W$ be the operator $w\left(-i \nabla_{z}\right)$ and let

$$
W^{1 / 2} X=\left\{f: W^{-1 / 2} f \in X\right\} .
$$

Any $f \in X$ is mapped by $B$ to $B f \in \mathscr{H}_{\sigma}$ and by $B_{\sigma}^{*} B$ to $B_{\sigma}^{*} B f \in X^{*}$. Correspondingly, $W^{1 / 2} f \in W^{1 / 2} X$ is mapped by $B$ to $W^{1 / 2} B f \in \mathscr{H}_{w^{-1} \sigma}$ and by $B_{w^{-1} \sigma}^{*} B=W^{-1} B_{\sigma}^{*} B$ to $W^{-1} W^{1 / 2} B_{\sigma}^{*} B f=W^{-1 / 2} B_{\sigma}^{*} B f \in W^{-1 / 2} X^{*}=\left(W^{1 / 2} X\right)^{*}$. Therefore if the pair $(\sigma, X)$ is admissible, so is also the pair $\left(w^{-1} \sigma, W^{1 / 2} X\right)$.

In the applications, one is often naturally provided with various admissible pairs $(\sigma, X)$ with different $\sigma$, thereby implementing the situation of Lemma 2.1 with different spaces $\mathscr{H}_{\sigma}$ and different adjoint operators $B_{\sigma}^{*}$. If all the measures under consideration are absolutely continuous with respect to a fixed measure $\sigma_{0}$, namely have the form $\sigma=w \sigma_{0}$ for a suitable $w$, one can take advantage of the previous invariance property to work with a fixed Hilbert space $\mathscr{H}=\mathscr{H}_{\sigma_{0}}$ 
and a fixed adjoint operator $B^{*}=B_{\sigma_{0}}^{*}$ by replacing the admissible pairs $(\sigma, X)$ by the admissible pairs $\left(\sigma_{0}, W^{1 / 2} X\right)$. Note however that for given $\sigma$ and $\sigma_{0}$, the function $w$ is defined at best on $\Sigma$ whereas it is required to be defined in $\mathbb{R}^{n+1}$ to allow for the definition of the operator $W$. In practical cases, we shall encounter a compelling criterion to remove that ambiguity (see next section).

The important Corollary 2.1 has an obvious analogue in the point of view of the Fourier transform.

Corollary 2.2. Let $\Sigma$ be as above and let $\left(\sigma_{i}, X_{i}\right), i=1,2$, be two admissible pairs such that $\sigma_{1}$ and $\sigma_{2}$ are absolutely continuous with respect to each other. Then the operator $\mathscr{F}^{-1}\left(\left(\sigma_{1} \sigma_{2}\right)^{1 / 2}\right) *$ is a bounded operator from $X_{1}$ to $X_{2}^{*}$ and from $X_{2}$ to $X_{1}^{*}$.

We now describe the first basic example of an admissible pair. That example will eventually provide a contact with the operator point of view. We assume that there exists an orthogonal decomposition $\mathbb{R}^{n+1}=\mathbb{R} \oplus \mathbb{R}^{n}$ (we denote by $z=(t, x)$ and $\zeta=(\eta, \xi)$ the corresponding decomposition of the generic variables) such that $\Sigma$ takes the form

$$
\Sigma=\{\zeta: \eta=\Phi(\xi)\},
$$

where $\Phi$ is a piecewise smooth function from an open subset $\Omega$ of $\mathbb{R}^{n}$ to $\mathbb{R}$ (one could assume without essential difficulty that $\Sigma$ is a finite union of such surfaces. See Remark 2.1 below for a related question). We can then use $\xi$ as a coordinate on $\Sigma$, identify $\mathscr{H}_{\sigma}$ with $L^{2}(\Omega, d \sigma(\xi))$ and rewrite (2.15), (2.16), and (2.17) as

$$
\begin{gathered}
B f(\xi)=(2 \pi)^{-(n+1) / 2} \int d t d x f(t, x) \exp (-i t \Phi(\xi)-i x \xi), \\
\left.\left(B_{\sigma}^{*} v\right)(t, x)=(2 \pi)^{-(n+1) / 2} \int d \sigma(\xi) v(\xi) \exp (i t \Phi(\xi)+i x \xi)\right), \\
\left(B_{\sigma}^{*} B f\right)(t, x)=(2 \pi)^{-(n+1)} \int d \sigma(\xi) d t^{\prime} d x^{\prime} f\left(t^{\prime}, x^{\prime}\right) \\
\times \exp \left[i\left(t-t^{\prime}\right) \Phi(\xi)+i\left(x-x^{\prime}\right) \xi\right] .
\end{gathered}
$$

In order to obtain the first class of examples, we assume in addition that there exists a suitably regular positive function $w$ defined on $\Sigma$ such that $w^{-1} \sigma$ projects onto the Lebesgue measure $d \xi$ in $\Omega$. Then the Plancherel theorem in the variables $(x, \xi)$ yields the relations

$$
\begin{gathered}
\left\|B f ; L^{2}\left(\Sigma, w^{-1} \sigma\right)\right\| \leq(2 \pi)^{-1 / 2}\left\|f ; L_{t}^{1}\left(\mathbb{R}, L_{x}^{2}\right)\right\| \\
\left\|B_{\sigma}^{*} v(t, \cdot) ; L_{x}^{2}\right\|=(2 \pi)^{-1 / 2}\left\|v w ; L^{2}\left(\Sigma, w^{-1} \sigma\right)\right\| \\
=(2 \pi)^{-1 / 2}\left\|v ; L^{2}(\Sigma, w \sigma)\right\| .
\end{gathered}
$$

In particular, $B$ is bounded from $L_{t}^{1}\left(\mathbb{R}, L_{x}^{2}\right)$ to $L^{2}\left(\Sigma, w^{-1} \sigma\right)$ and $B_{\sigma}^{*}$ from $L^{2}(\Sigma, \sigma)$ to $L_{t}^{\infty}\left(\mathbb{R}, L_{x}^{2}\right)$. We can now use the invariance property to obtain admissible pairs: we extend $w$ from $\Sigma$ to $\mathbb{R}^{n+1}$, define again $W=w\left(-i \nabla_{z}\right)$ and obtain the admissible pairs $\left(\sigma, X=W^{-1 / 2} L_{t}^{1}\left(\mathbb{R}, L_{x}^{2}\right)\right)$ for that particular $\sigma$.

If on the other hand we assume that $\Omega=\mathbb{R}^{n}$, then we can make contact between the operator and Fourier transform points of view. We define the operator

$$
L=i \Phi\left(-i \nabla_{x}\right)
$$

and the unitary group

$$
U(t)=\exp \left(i t \Phi\left(-i \nabla_{x}\right)\right)
$$


Then for all $f \in \mathscr{S}_{n+1}$

$$
B f=(2 \pi)^{-1 / 2} \mathscr{F}_{n} \int d \tau U(-\tau) f(\tau)=(2 \pi)^{-1 / 2} \mathscr{F}_{n} A f,
$$

where $A$ is defined by (2.5).

If we assume both that there exists a $w$ as above and that $\Omega=\mathbb{R}^{n}$, then the previous example becomes a special case of the basic example in the operator point of view. In fact, in addition to (2.27), we have now

$$
B_{\sigma}^{*} v=(2 \pi)^{-1 / 2} U(t) \mathscr{F}_{n}^{-1} w v=(2 \pi)^{-1 / 2} A^{*} \mathscr{F}_{n}^{-1} w v .
$$

We extend $w$ from $\Sigma$ to $\mathbb{R}^{n+1}$ by taking the (unique) extension which is independent of $\eta$, so that the previous $X$ becomes

$$
X=W^{-1 / 2} L_{t}^{1}\left(\mathbb{R}, L_{x}^{2}\right)=L_{t}^{1}\left(\mathbb{R}, W^{-1 / 2} L_{x}^{2}\right)
$$

and we obtain finally the following identifications:

$$
\begin{array}{ccc}
X=L_{t}^{1}(\mathbb{R}, \mathscr{H}) & L^{2}(\Sigma, \sigma) & \stackrel{B_{\sigma}^{*}}{\longrightarrow} X^{*}=L_{t}^{\infty}\left(\mathbb{R}, W^{1 / 2} L_{x}^{2}\right) \\
\uparrow_{\mathscr{F}_{n}} & \uparrow_{W} \\
\mathscr{H}=W^{-1 / 2} L_{x}^{2} \stackrel{A^{*}}{\longrightarrow} & L_{t}^{\infty}(\mathbb{R}, \mathscr{H})
\end{array}
$$

The fact that the final spaces are different in the upper and lower parts of the diagram is due to the fact that the adjoint operators $B_{\sigma}^{*}$ and $A^{*}$ are defined with the dualities of $L^{2}\left(\mathbb{R}^{n+1}\right)$ and $L^{2}(\mathbb{R}, \mathscr{H})$ respectively.

The previous correspondence extends in an obvious way to the case of general admissible pairs $(\sigma, X)$, where $X$ is not necessarily of the form (2.29), and provides the translation of the admissibility of $(\sigma, X)$ into properties of $A$, which are of direct relevance for the equation (1.1). In fact, let $(\sigma, X)$ be an admissible pair such that there exists $w$ defined on $\Sigma$ with $w^{-1} \sigma$ projecting onto the Lebesgue measure $d \xi$. We extend $w$ to $\mathbb{R}^{n+1}$ as a function of $\xi$ only and define again $W=w\left(-i \nabla_{z}\right)$. We then obtain the diagram

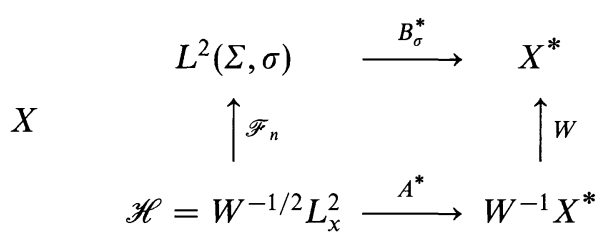

In particular, $A^{*}$ and $A^{*} A$ are bounded operators from $W^{-1 / 2} L_{x}^{2}$ and from $X$ respectively to $W^{-1} X^{*}$. This suggests that the equation (1.1) in the form (1.2) should be solved in the space $W^{-1} X^{*}$ (or in an intersection of such spaces associated with the same $\sigma$ ) for initial data $u_{0} \in \mathscr{H}=W^{-1 / 2} L_{x}^{2}$ and with $f \in X$. Note also that the invariance seen above for the admissible pairs $(\sigma, X)$ is translated, in the operator formalism, into the obvious invariance under the replacement of $\mathscr{H}$ by $S \mathscr{H}$, where $S$ is an operator commuting with $U(\cdot)$, for instance of $L_{x}^{2}$ by $W^{-1 / 2} L_{x}^{2}$. In the study of the Cauchy problem for the equation (1.1), $\mathscr{H}$ is the space of initial data $u_{0}$, and that invariance is important in allowing for some flexibility in the choice of that space. An example of special interest is the case where $w(\xi)=\left(1+\xi^{2}\right)^{s}, s \in \mathbb{R}$, where $\mathscr{H}=W^{-1 / 2} L_{x}^{1}$ is the usual Sobolev space $H^{s}$. 
We now turn to a second class of examples which is obtained by generalizing the spaces $X_{r}$ that occur in the case of the Schrödinger equation. In that special case, they are readily obtained from the explicit representation (2.8). In more general situations, they will be obtained from (2.14), from space time estimates of $\mathscr{F}^{-1}(\sigma)$ and from suitable inequalities such as the Young or HLS inequalities. The case of the Schrödinger equation is especially simple in that respect for two reasons: (i) the optimal space-time estimates are naturally obtained for the measure $d \sigma(\xi)=d \xi$ for which the operators $\left(A, A^{*}\right)$ coincide with the operators $\left(B, B_{\sigma}^{*}\right)$, namely $w=1$ in the diagram $(2.31)$, and (ii) $\mathscr{F}^{-1}(\sigma)$ can be computed explicitly, thereby yielding the estimate (2.9) immediately. In general (i) it will be necessary to consider various choices of $\sigma$ and (ii) the explicit computation of $\mathscr{F}^{-1}(\sigma)$ will have to be replaced by stationary phase estimates. We now describe a class of examples in space dimension $n=1$ [15], which illustrate that situation and fit nicely into the general formalism. The basic ingredient consists of estimates of $\mathscr{F}^{-1}(\sigma)$ in space-time, as mentioned above. Those estimates are obtained by the method of stationary phase. The basic technical result is the following [15].

Proposition 2.1. Let $\Omega \subset \mathbb{R}$ be a finite union of open intervals, let $\Phi_{0} \in \mathscr{C}^{2}(\Omega)$, $\Phi_{0}$ real valued with $\Phi_{0}^{\prime \prime} \neq 0$. Assume that $\Phi_{0}^{\prime \prime}$ has bounded variation in all compact subsets of $\Omega$ and behaves as a power in a neighborhood of $\partial \Omega$ in the following sense: for any $\xi_{*} \in \partial \Omega$ there exists $\beta \in \mathbb{R}, \beta \neq 0$ and $c_{1}, c_{2}, c_{3}>0$ such that for $\xi \in \Omega$, $\xi$ in a neighborhood of $\xi_{*}$, the following inequalities hold:

$$
\begin{array}{r}
c_{1}\left|\xi-\xi_{*}\right|^{\beta-2} \leq\left|\Phi_{0}^{\prime \prime}(\xi)\right| \leq c_{2}\left|\xi-\xi_{*}\right|^{\beta-2}, \\
\left.\left|\int_{\xi *+\delta}^{\xi_{*}+2 \delta}\right| d \Phi_{0}^{\prime \prime}(\xi)\left|\leq c_{3}\right| \delta\right|^{\beta-2}
\end{array}
$$

if $\left|\xi_{*}\right|<\infty$, and

$$
\begin{gathered}
c_{1}|\xi|^{\beta-2} \leq\left|\Phi_{0}^{\prime \prime}(\xi)\right| \leq c_{2}|\xi|^{\beta-2}, \\
\left|\int_{\xi}^{2 \xi}\right| d \Phi_{0}^{\prime \prime}\left(\xi^{\prime}\right)|| \leq c_{3}|\xi|^{\beta-2}
\end{gathered}
$$

if $\xi_{*}= \pm \infty$. (the exponent $\beta$ may depend on the point $\xi_{*}$ ).

Then for all $t \neq 0$

$$
\left.\left.\left|\int_{\Omega} d \xi \exp \left[i t \Phi_{0}(\xi)\right]\right| \Phi_{0}^{\prime \prime}(\xi)\right|^{1 / 2}\left|\leq C_{\Phi_{0}}\right| t\right|^{-1 / 2},
$$

where $C_{\Phi_{0}}$ depend on $\Phi_{0}$ through $\Phi_{0}^{\prime \prime}$ only.

For completeness, and also as a preparation to the proof of the more general Proposition 3.3 below, a proof of that proposition is given in the Appendix.

Proposition 2.1 yields a second example of an admissible pair, and by interpolation with the first example, a one parameter family of such pairs [15].

Proposition 2.2. Let $\Phi$ satisfy the assumptions made on $\Phi_{0}$ in Proposition 2.1. Then the following pairs are admissible:

$$
d \sigma(\xi)=d \xi, \quad X=L_{t}^{1}\left(\mathbb{R}, L_{x}^{2}\right)\left(X^{*}=L_{t}^{\infty}\left(\mathbb{R}, L_{x}^{2}\right)\right),
$$




$$
d \sigma(\xi)=\left|\Phi^{\prime \prime}(\xi)\right|^{1 / 2} d \xi, \quad X=L_{t}^{4 / 3}\left(\mathbb{R}, L_{x}^{1}\right)\left(X^{*}=L_{t}^{4}\left(\mathbb{R}, L_{x}^{\infty}\right)\right),
$$

and by interpolation

$$
d \sigma(\xi)=\left|\Phi^{\prime \prime}(\xi)\right|^{\alpha(r)} d \xi, \quad X=L_{t}^{\bar{q}}\left(\mathbb{R}, L_{x}^{\bar{r}}\right)\left(X^{*}=L_{t}^{q}\left(\mathbb{R}, L_{x}^{r}\right)\right)
$$

with $2 / q=\alpha(r)=1 / 2-1 / r, 2 \leq r \leq \infty$.

Proof. The first case has already been established. The second case follows from (2.14) and Proposition 2.1 via the condition (3)of Lemma 2.1. In fact

$$
\mathscr{F}^{-1} \sigma=(2 \pi)^{-1} \int d \sigma(\xi) \exp (i t \Phi(\xi)+i x \xi) .
$$

By Proposition 2.1 with $\Phi_{0}=\Phi+x \xi / t$, we estimate

$$
\left|\mathscr{F}^{-1} \sigma\right| \leq C_{\Phi}|t|^{-1 / 2}
$$

uniformly in $x$, since $C_{\Phi}$ depends only on $\Phi_{0}^{\prime \prime}=\Phi^{\prime \prime}$, and the result follows from the HLS inequality [11] in the time variable. QED

In the point of view of the Fourier transform, one starts with a surface $\Sigma \subset \mathbb{R}^{n+1}$ without making reference to a specific decomposition $\mathbb{R}^{n+1}=\mathbb{R} \oplus \mathbb{R}^{n}$. One may therefore expect to obtain different examples of admissible pairs by applying the same methods to various such decompositions, in so far as $\Sigma$ can be decomposed as a finite union of pieces described by functions $\Phi$ satisfying the assumptions of Proposition 2.1. In space time dimension $1+1$, a case of special interest is obtained by exchanging $\xi$ and $\eta$ (or equivalently $x$ and $t$ ). That case has been considered in [15]. We shall state the results in the special case where $\Phi$ is a bijection from $\mathbb{R}$ to $\mathbb{R}$, which is relevant for the generalized Benjamin-Ono equation with $\Phi(\xi)=\xi|\xi|^{2 \mu}, \mu>0$. We shall indicate briefly in Remark 2.1 below the modifications that would be necessary to treat more general cases, covering in particular the Schrödinger equation with $\Phi(\xi)=\xi^{2}$ and more general equations with $\Phi(\xi)=|\xi|^{2 \mu}, \mu>0$.

Let $\Psi$ be the inverse function to $\Phi$, namely $\Phi \circ \Psi=\Psi \circ \Phi=1$. One can then rewrite $B_{\sigma}^{*}$ given by (2.21) with $n=1$ as

$$
B_{\sigma}^{*} v(t, x)=(2 \pi)^{-1} \int d \sigma(\Psi(\eta)) v(\Psi(\eta)) \exp [i t \eta+i x \Psi(\eta)]
$$

In the special case $d \sigma(\xi)=d \eta$, the Plancherel theorem in the time variable gives

$$
\left\|B_{\sigma}^{*} v(\cdot, x) ; L_{t}^{2}\right\|^{2}=(2 \pi)^{-1} \int d \eta|v(\Psi(\eta))|^{2}=(2 \pi)^{-1} \int d \sigma(\xi)|v(\xi)|^{2},
$$

thereby yielding (via the second condition of Lemma 2.1) the admissible pair $\left(d \sigma(\xi)=d \eta=\left|\Phi^{\prime}(\xi)\right| d \xi, X=L_{x}^{1}\left(\mathbb{R}, L_{t}^{1}\right)\right)$. Since we have assumed $\Phi$ and $\Psi$ to be bijections of $\mathbb{R}$, we could recast that example in a form similar to (2.5), (2.6), (2.7) by introducing the unitary one parameter group

$$
V(x)=\exp \left[i x \Psi\left(-i \partial_{t}\right)\right]
$$

in $L_{t}^{2}(\mathbb{R})$, describing the propagation in the $x$ direction of functions of $t$. However that group does not play any interesting role in solving the Cauchy problem for the equation (1.1).

One can also apply Proposition 2.1 in the permuted situation, namely estimate

$$
\mathscr{F}^{-1}(\sigma)=(2 \pi)^{-1} \int d \sigma(\Psi(\eta)) \exp [i t \eta+i x \Psi(\eta)] .
$$


In particular, if $\Psi$ satisfies the assumptions made on $\Phi_{0}$ in Proposition 2.1 and if one chooses $d \sigma(\xi)=\left|\Psi^{\prime \prime}(\eta)\right|^{1 / 2} d \eta$, then

$$
\left|\mathscr{F}^{-1}(\sigma)\right| \leq C|x|^{-1 / 2}
$$

uniformly in $t$, which in the same way as in Proposition 2.2 yields the admissible pair $\left(\sigma, X=L_{x}^{4 / 3}\left(\mathbb{R}, L_{t}^{1}\right)\right)$ for that $\sigma$.

These remarks lead immediately to the following proposition [15].

Proposition 2.3. Let $\Phi \circ \Psi=\Psi \circ \Phi=1$ and let $\Psi$ satisfy the assumptions made on $\Phi_{0}$ in Proposition 2.1. Then the following pairs are admissible

$$
\begin{gathered}
d \sigma(\xi)=d \eta=\left|\Phi^{\prime}(\xi)\right| d \xi, \quad X=L_{x}^{1}\left(\mathbb{R}, L_{t}^{2}\right)\left(X^{*}=L_{x}^{\infty}\left(\mathbb{R}, L_{t}^{2}\right)\right) \\
d \sigma(\xi)=\left|\Psi^{\prime \prime}(\eta)\right|^{1 / 2} d \eta=\left|\Phi^{\prime \prime}(\xi) / \Phi^{\prime}(\xi)\right|^{1 / 2} d \xi \\
X=L_{x}^{4 / 3}\left(\mathbb{R}, L_{t}^{1}\right)\left(X^{*}=L_{x}^{4}\left(\mathbb{R}, L_{t}^{\infty}\right)\right)
\end{gathered}
$$

and by interpolation

$$
\begin{gathered}
d \sigma(\xi)=\left|\Phi^{\prime \prime}(\xi)\right|^{\alpha(r)}\left|\Phi^{\prime}(\xi)\right|^{1-3 \alpha(r)} d \xi \\
X=L_{x}^{\bar{q}}\left(\mathbb{R}, L_{t}^{\bar{r}}\right)\left(X^{*}=L_{x}^{q}\left(\mathbb{R}, L_{t}^{r}\right)\right)
\end{gathered}
$$

with $2 / q=\alpha(r)=1 / 2-1 / r, 2 \leq r \leq \infty$.

The proof has been already given except for the elementary computation of

$$
\Psi^{\prime \prime}(\eta)=-\Phi^{\prime}(\xi)^{-3} \Phi^{\prime \prime}(\xi)
$$

Note also that the families of admissible pairs of Propositions 2.2 and 2.3 have a common member, namely $\left(d \sigma(\xi)=\left|\Phi^{\prime \prime}(\xi)\right|^{1 / 3} d \xi, X=L_{x \times t}^{6 / 5}\left(\mathbb{R}^{2}\right)\right)$.

Remark 2.1. The extension of Proposition 2.3 to the case where $\Phi$ is not one to one from $\mathbb{R}$ to $\mathbb{R}$ is easy. We assume that $\mathbb{R}=\bigcup_{j} I_{j}$ is the union of a finite number of intervals where $\Phi$ is monotonous. Each $I_{j}$ is mapped in a one to one fashion on an interval $J_{j}$. Let $\Psi_{j}$ be the inverse map from $J_{j}$ to $I_{j}$. Then, for instance

$$
\begin{aligned}
B_{\sigma}^{*} v(t, x) & =\sum_{j} B_{\sigma, j}^{*} v(t, x) \\
& =\sum_{j}(2 \pi)^{-1} \int d \sigma_{j}\left(\Psi_{j}(\eta)\right) v\left(\Psi_{j}(\eta)\right) \exp \left[i t \eta+i x \Psi_{j}(\eta)\right]
\end{aligned}
$$

where $d \sigma_{j}(\xi)=\chi_{I_{j}}(\xi) d \sigma(\xi)$. One can then reconstruct estimates similar to those of Proposition 2.3. For instance if $d \sigma_{j}(\xi)=\chi_{J_{j}}(\eta) d \eta$, the Plancherel theorem in the time variable gives

$$
\int d t\left|B_{\sigma, j}^{*} v(t, x)\right|^{2}=(2 \pi)^{-1} \int d \eta \chi_{J_{j}}(\eta)\left|v\left(\Psi_{j}(\eta)\right)\right|^{2}
$$

and therefore

$$
\left\|B_{\sigma}^{*} v ; L_{t}^{2}\right\|^{2} \leq N(2 \pi)^{-1} \sum_{j} \int d \eta \chi_{J_{j}}(\eta)\left|v\left(\Psi_{j}(\eta)\right)\right|^{2},
$$

where $N$ is the number of terms in the sum (2.49). Of special interest is the case where $\Phi$ is an even function which maps $\mathbb{R}^{+}$onto $\mathbb{R}^{+}$in a one to one way, for 
instance $\Phi(\xi)=|\xi|^{2 \mu}$. With $\Psi$ the inverse map from $\mathbb{R}^{+}$to $\mathbb{R}^{+}$and $\sigma$ an even measure, one obtains from (2.49)

$$
B_{\sigma}^{*} v(t, x)=\sum_{ \pm}(2 \pi)^{-1} \int d \sigma(\Psi(\eta)) v( \pm \Psi(n)) \exp [i t \eta \pm i x \Psi(\eta)]
$$

and for $d \sigma(\Psi(\eta))=d \eta$

$$
\left\|B_{\sigma}^{*} ; L_{t}^{2}\right\|^{2} \leq 2(2 \pi)^{-1} \int_{0}^{\infty} d \eta \sum_{ \pm}|v( \pm \Psi(\eta))|^{2} .
$$

The estimates based on Proposition 2.1 via (2.45) extend in an obvious way to the present situation and one obtains the same family of admissible pairs as in Proposition 2.3, with the same spaces $X$ and associated measures which are piecewise as indicated.

We finally mention the case of the wave equation in $\mathbb{R}^{n}$

$$
\partial_{t}^{2} u-\Delta u=f
$$

for which the Cauchy problem with initial data $u(0)=u_{0}, \partial_{t} u(0)=v_{0}$ is equivalent to the integral equation

$$
u(t)=(\cos \omega t) u_{0}+\omega^{-1}(\sin \omega t) v_{0}+\int_{0}^{t} d \tau \omega^{-1} \sin \omega(t-\tau) f(\tau),
$$

where $\omega=(-\Delta)^{1 / 2}$. The relevant unitary group in that case is $U(t)=\exp (i \omega t)$ and the relevant surface $\Sigma$ is defined by $\Phi(\xi)=|\xi|$. The known space time integrability properties associated with that equation $[3,8,19,21,22]$ are naturally obtained in the form of the admissibility of the pairs

$$
\left(d \sigma(\xi)=|\xi|^{-(n+1) \alpha(r)} d \xi, X=L_{t}^{\bar{q}}\left(\mathbb{R} ; \dot{B}_{\bar{r}, 2}^{0}\right)\right)
$$

with $0 \leq 2 / 9=(n-1) \alpha(r)<1$. Here $\dot{B}_{\bar{r}, 2}^{0}$ are homogeneous Besov spaces (see [2] for general information and [8], appendix for a summary and for the notation). Similar but more complicated results hold for the Klein-Gordon equation [4], see also [9]. The proofs are a combination of dyadic decompositions and stationary phase estimates.

\section{Retarded Estimates, Applications}

We have seen in the previous section that the existence of admissible pairs $(\sigma, X)$ yields a natural framework to solve the equation (1.1) in the form (1.2). In particular the diagram (2.31) suggests to look for solutions in $W^{-1} X^{*}$ for initial data $u_{0} \in W^{-1 / 2} L_{x}^{2}$ and $f \in X$. In that discussion however, we have overlooked the fact that the time integral in (1.2) is restricted to $0 \leq \tau \leq t$. The restriction $\tau \geq 0$ is relatively harmless since it can be incorporated into the definition of $f$ as a support condition in time. That restriction does not spoil the basic estimates in so far as the multiplication by a sharp cut-off function in time is a bounded operator in $X$. We shall say that $X$ is time cut-off stable if it possesses that property uniformly in the cut-off time. It is important for the applications to look for admissible pairs $(\sigma, X)$ for which that property holds. 
We have seen in the previous section that the admissibility of $(\sigma, X)$ is preserved by the change $\sigma \rightarrow w^{-1} \sigma, X \rightarrow W^{1 / 2} X$ for an arbitrary positive suitably regular function $w$ defined in $\mathbb{R}^{n+1}$, a property that is useful to reduce various admissible pairs to the case of a single measure $\sigma_{0}$ on $\Sigma$. We now have a compelling criterion to remove the ambiguity of extending $w$ from $\Sigma$ to $\mathbb{R}^{n+1}$ which occurs in that reduction, namely the requirement that time cut-off stability be preserved: if $X$ is time cut-off stable, that condition imposes to extend $w$ as a function of $\xi$ only, so that $W$ does not contain time derivatives in its definition.

We now concentrate on the second restriction $\tau \leq t$, which is much more serious. It replaces the operator $A^{*} A$ by the retarded operator

$$
\left(A^{*} A\right)_{R} f(t)=\int d \tau U(t-\tau) \theta(t-\tau) f(\tau),
$$

where $\theta$ is the Heaviside function, and similarly $B_{\sigma}^{*} B$ by the retarded operator (cf. (2.14))

$$
\left(B_{\sigma}^{*} B\right)_{R}=(2 \pi)^{-(n+1) / 2}\left(\theta \mathscr{F}^{-1}(\sigma)\right) * .
$$

The retardation breaks the factorisation of $A^{*} A$ and $B_{\sigma}^{*} B$ as a product and therefore makes Lemma 2.1 inapplicable. This leaves open the question whether the estimates obtained for $A^{*} A$ and $B_{\sigma}^{*} B$ in the previous framework still hold for the retarded operators. That question occurs both for the diagonal estimates from $X$ to $X^{*}$ and for the non-diagonal estimates from $X_{1}$ to $X_{2}^{*}$ that appear in Corollaries 2.1 and 2.2. The present section is devoted to that problem. We shall first describe a few cases, already widely used in the literature, where the estimates obviously extend to the retarded operators, and then consider nontrivial situations associated with the special case covered by Propositions 2.2 and 2.3.

The first obvious situation is that where one of the spaces is of the type $L_{t}^{1}(\mathbb{R}, \mathscr{H})$ and is best described in the operator point of view.

Proposition 3.1. Let $\mathscr{H}$ be a Hilbert space, $X \subset \mathscr{S}_{n+1}^{\prime}$ a Banach space, let $X$ be time cut-off stable, and let the conditions of Lemma 2.1 hold with $T=A$ defined by (2.5). Then the operator $\left(A^{*} A\right)_{R}$ is (strictly speaking extends to) a bounded operator from $L_{t}^{1}(\mathbb{R}, \mathscr{H})$ to $X^{*}$ and from $X$ to $L_{t}^{\infty}(\mathbb{R}, \mathscr{H})$.

Proof. We prove the second property, from which the first one follows by duality. Let $f \in \mathscr{D}$. Then for each $t$,

$$
\begin{aligned}
\left\|\left(A^{*} A\right)_{R} f(t) ; \mathscr{H}\right\| & =\|A \theta(t-\cdot) f ; \mathscr{H}\| \\
& \leq a\left\{\operatorname{Sup}_{t}\|\theta(t-\cdot) ; B(X)\|\right\}\|f ; X\|
\end{aligned}
$$

by the unitarity of $U$ in $\mathscr{H}$, the estimate (2.1), and the time cut-off stability of $X$. QED

The second obvious situation where retardation preserves the estimates, and which we refrain from formalizing, is that where the boundedness of $A^{*} A$ or $B_{\sigma}^{*} B$ follows from space-time estimates of $\mathscr{F}^{-1}(\sigma)$ such as $(2.41)$ or $(2.45)$ or from time estimates of space norms such as (2.9).

The previous two situations cover the cases of all the spaces associated with the Schrödinger equation in Sect.2. In particular the estimates (2.11) extend to the retarded operators. The same situation prevails for all the estimates associated with the admissible pairs (2.53) in the case of the wave equation. In both cases, 
the time cut-off stability of the spaces $X$ follows immediately from the fact that time occurs in the form of an $L^{\bar{q}}$ condition with no derivatives.

We next consider in more detail the case covered by Propositions 2.2 and 2.3. We take $n=1$ and define $L$ and $U$ by $(2.25),(2-26)$ for some $\Phi$ satisfying the assumptions of Proposition 2.1 with $\bar{\Omega}=\mathbb{R}$ and the assumptions of Proposition 2.3. For definiteness, we use the notation $X_{1}=L_{t}^{1}\left(\mathbb{R}, L_{x}^{2}\right), X_{2}=L_{x}^{1}\left(\mathbb{R}, L_{t}^{2}\right), X_{3}=$ $L_{t}^{4 / 3}\left(\mathbb{R}, L_{x}^{1}\right), X_{4}=L_{x}^{4 / 3}\left(\mathbb{R}, L_{t}^{1}\right)$ and similarly $d \sigma_{1}=d \xi, d \sigma_{2}=d \eta, d \sigma_{3}=\left|\Phi^{\prime \prime}\right|^{1 / 2} d \xi$, $d \sigma_{4}=\left|\Psi^{\prime \prime}\right|^{1 / 2} d \eta$ for the associated measures. Proposition 3.1 shows that the retarded estimates hold from $X_{1}$ to $X_{i}^{*}$ (and from $X_{i}$ to $X_{1}^{*}$ ) for all $i, 1 \leq i \leq 4$. The second obvious situation ensures the retarded estimates from $X_{3}$ to $X_{3}^{*}$ and from $X_{4}$ to $X_{4}^{*}$.

We now turn to the less obvious cases, beginning with a study of the retarded operators from $X_{i}$ to $X_{2}^{*}$ for $i=2,3,4$. We recall that $\Phi$ is assumed to be a bijection from $\mathbb{R}$ to $\mathbb{R}$ and that the measures $\sigma_{i}, i=2,3,4$ are absolutely continuous with one another. We denote by ${ }^{\wedge}$ the Fourier transform with respect to $t$, we recall that

$$
\theta(t)=(2 \pi i)^{-1} \int d \eta(\eta-i 0)^{-1} \exp (i t \eta)
$$

and we introduce the functions

$$
\begin{aligned}
& h_{2}(\eta, x)=(2 \pi i)^{-1} \int d \sigma_{2}\left(\xi^{\prime}\right)\left(\eta^{\prime}-\eta-i 0\right)^{-1} \exp \left(i x \xi^{\prime}\right), \\
& h_{i}(\eta, x)=(2 \pi i)^{-1}\left(d \sigma_{i}(\xi) / d \sigma_{2}(\xi)\right)^{-1 / 2} \\
& \times \int d \tilde{\sigma}_{i}\left(\xi^{\prime}\right)\left(\eta^{\prime}-\eta-i 0\right)^{-1} \exp \left[i x\left(\xi^{\prime}-\xi\right)\right], \\
& K_{i}\left(t ; x, x^{\prime}\right)=\int d \sigma_{i}(\xi) h_{i}(\eta, x) \exp \left[i t \eta+i\left(x-x^{\prime}\right) \xi\right]
\end{aligned}
$$

for $i=3,4$, where the auxiliary measures $\tilde{\sigma}_{i}$ are defined by $\tilde{\sigma}_{i}=\left(\sigma_{i} \sigma_{2}\right)^{1 / 2}$. Here and below it is understood that $\eta=\Phi(\xi), \eta^{\prime}=\Phi\left(\xi^{\prime}\right)$. By Corollary 2.2, the properties of interest are the boundedness properties of the operators $\left(B_{\tilde{\sigma}_{t}}^{*} B\right)_{R}$ from $X_{i}$ to $X_{2}^{*}$ and from $X_{2}$ to $X_{i}^{*}$ for $i=2,3,4$. We first proceed to an algebraic reduction of the problem.

Proposition 3.2. Let $f, g \in \mathscr{S}_{2}$. Then

(1) The following identity holds

$$
\left\langle g,\left(B_{\sigma_{2}}^{*} B\right)_{R} f\right\rangle=(2 \pi)^{-1} \int d x d x^{\prime} d \eta \overline{\hat{g}}(\eta, f) \hat{f}\left(\eta, x^{\prime}\right) \bar{h}_{2}\left(\eta, x^{\prime}-x\right) .
$$

A sufficient condition for $\left(B_{\sigma_{2}}^{*} B\right)_{R}$ to be bounded from $X_{2}$ to $X_{2}^{*}$ is that $h_{2} \in L^{\infty}\left(\mathbb{R}^{2}\right)$. Then

$$
\left\|\left(B_{\sigma_{2}}^{*} B\right)_{R} f ; X_{2}^{*}\right\| \leq(2 \pi)^{-1}\left\|h_{2}\right\|_{\infty}\left\|f ; X_{2}\right\| .
$$

(2) The following identity holds for $i=3,4$

$$
\begin{aligned}
\int d \tau\left|\left(B_{\tilde{\sigma}_{l}}^{*} B\right)_{R} f(\tau, y)\right|^{2}= & (2 \pi)^{-3} \int d t d x d t^{\prime} d x^{\prime} \bar{f}(t, x) f\left(t^{\prime}, x^{\prime}\right) \\
& \times\left\{\theta\left(t-t^{\prime}\right) K_{i}\left(t-t^{\prime} ; x-y, x^{\prime}-y\right)\right. \\
& \left.+\theta\left(t^{\prime}-t\right) \bar{K}_{i}\left(t^{\prime}-t ; x^{\prime}-y, x-y\right)\right\} .
\end{aligned}
$$

$A$ sufficient condition for $\left(B_{\tilde{\sigma}_{3}}^{*} B\right)_{R}$ to be bounded from $X_{3}$ to $X_{2}^{*}$ and from $X_{2}$ to $X_{3}^{*}$ is that $K_{3}$ satisfies the estimate

$$
\left|K_{3}\left(t ; x, x^{\prime}\right)\right| \leq C|t|^{-1 / 2} .
$$


$A$ sufficient condition for $\left(B_{\tilde{\sigma}_{4}}^{*} B\right)_{R}$ to be bounded from $X_{4}$ to $X_{2}^{*}$ and from $X_{2}$ to $X_{4}^{*}$ is that $K_{4}$ satisfies the estimate

$$
\left|K_{4}\left(t ; x, x^{\prime}\right)\right| \leq C\left|x-x^{\prime}\right|^{-1 / 2} .
$$

Proof. Part (1). We introduce the representation (3.4) of $\theta$ in the definition $\left(B_{\sigma_{2}}^{*} B\right)_{R}$ and obtain

$$
\begin{aligned}
\left\langle g,\left(B_{\sigma_{2}}^{*} B\right)_{R} f\right\rangle= & (2 \pi)^{-2} \int d t d x d t^{\prime} d x^{\prime} \bar{g}(t, x) f\left(t^{\prime}, x^{\prime}\right) \\
& \times \int d \sigma_{2}\left(\xi^{\prime}\right) \exp \left[i\left(t-t^{\prime}\right) \eta^{\prime}+i\left(x-x^{\prime}\right) \xi^{\prime}\right](2 \pi i)^{-1} \\
& \times \int d \eta\left(\eta-\eta^{\prime}-i 0\right)^{-1} \exp \left[i\left(\eta-\eta^{\prime}\right)\left(t-t^{\prime}\right)\right] .
\end{aligned}
$$

Integration over $t, t^{\prime}$ and comparison with (3.5) yields (3.8) immediately. The subsequent statement as well as (3.9) follow directly from (3.8).

Part (2). We compute

$$
\begin{aligned}
\int d \tau\left|\left(B_{\tilde{\sigma}_{i}}^{*} B\right)_{R} f(\tau, y)\right|^{2}= & (2 \pi)^{-4} \int d t d x d t^{\prime} d x^{\prime} \bar{f}(t, x) f\left(t^{\prime}, x^{\prime}\right) \\
& \times \int d \tilde{\sigma}_{i}(\xi) d \tilde{\sigma}_{i}\left(\xi^{\prime}\right) \exp \left[i\left(y-x^{\prime}\right) \xi^{\prime}-i(y-x) \xi\right] \\
& \times \int d \tau \theta(\tau-t) \theta\left(\tau-t^{\prime}\right) \exp \left[i\left(\tau-t^{\prime}\right) \eta^{\prime}-i(\tau-t) \eta\right] .
\end{aligned}
$$

The last integral is explicitly computed to be

$$
i^{-1}\left(\eta-\eta^{\prime}-i 0\right)^{-1}\left\{\theta\left(t-t^{\prime}\right) \exp \left[i\left(t-t^{\prime}\right) \eta^{\prime}\right]+\theta\left(t^{\prime}-t\right) \exp \left[i\left(t-t^{\prime}\right) \eta\right]\right\},
$$

so that (3.13) reduces to (3.10) with

$$
\begin{aligned}
K_{i}\left(t-t^{\prime} ; x-y, x^{\prime}-y\right)= & \int d \tilde{\sigma}_{i}(\xi) d \tilde{\sigma}_{i}\left(\xi^{\prime}\right)(2 \pi i)^{-1}\left(\eta-\eta^{\prime}-i 0\right)^{-1} \\
& \times \exp \left[i\left(y-x^{\prime}\right) \xi^{\prime}-i(y-x) \xi+i\left(t-t^{\prime}\right) \eta^{\prime}\right]
\end{aligned}
$$

which is readily seen to coincide with (3.6), (3.7) by exchanging $(\xi, \eta)$ and $\left(\xi^{\prime}, \eta^{\prime}\right)$.

The subsequent boundedness statements follow immediately from (3.10), from the HLS inequality and from (3.11), (3.12) respectively. QED

Remark 3.1. If one replaces $\left[2 \pi i\left(\eta^{\prime}-\eta-i 0\right)\right]^{-1}$ by $\delta\left(\eta-\eta^{\prime}\right)$, one recovers the estimates of the previous section for the operators without retardation. With those estimates already available, it is therefore equivalent to establish the new ones with an $i 0$ prescription or a principal value prescription for the $\left(\eta^{\prime}-\eta\right)$ denominators.

In the framework of Proposition 2.1, the proof of the estimates (3.11) and (3.12) can be further reduced to that of estimates of the functions $h_{i}, i=3,4$, by means of the following result.

Proposition 3.3. Let $\Phi_{0}$ satisfy the assumptions of Proposition 2.1. Let $h \in\left(\mathscr{C}^{1} \cap\right.$ $\left.L^{\infty}\right)(\Omega)$ and assume that for each $\xi_{*} \in \partial \Omega, h$ satisfies

$$
\left(\xi-\xi_{*}\right) h^{\prime}(\xi) \in L^{\infty}\left(V\left(\xi_{*}\right)\right)
$$

for some neighborhood $V\left(\xi_{*}\right)$ of $\xi_{*}$, if $\xi_{*} \neq \pm \infty$, and

$$
\xi h^{\prime}(\xi) \in L^{\infty}\left(V_{ \pm \infty}\right)
$$

if $\xi_{*}= \pm \infty$. Then

$$
\left.\left.\left|\int_{\Omega} d \xi \exp \left[i t \Phi_{0}(\xi)\right]\right| \Phi_{0}^{\prime \prime}(\xi)\right|^{1 / 2} h(\xi)\left|\leq C_{\Phi_{0}, h}\right| t\right|^{-1 / 2}
$$


where $C_{\Phi_{0}, h}$ depends on $\Phi_{0}$ through $\Phi_{0}^{\prime \prime}$ only, and depends on $h$ through the norms $\left\|h ; L^{\infty}(\Omega)\right\|,\left\|\left(\xi-\xi_{*}\right) h^{\prime}(\xi) ; L^{\infty}\left(V\left(\xi_{*}\right)\right)\right\|,\left\|\xi h^{\prime}(\xi) ; L^{\infty}\left(V_{ \pm \infty}\right)\right\|$ if $\pm \infty \in \partial \Omega$, and $\left\|h^{\prime} ; L^{\infty}(K)\right\|$ only, where

$$
K=\Omega \backslash \bigcup_{\xi_{*} \in \partial \Omega} V\left(\xi_{*}\right) .
$$

The proof is a minor variation of that of Proposition 2.1 and is given in the Appendix, following the latter.

In order to give a relatively explicit class of examples where the previous functions $h_{i}$ satisfy the assumptions of Proposition 3.3, we now restrict our attention to the case where

$$
\Phi(\xi)=\xi|\xi|^{2 \mu}=\operatorname{sgn} \xi|\xi|^{\beta}
$$

with $\mu>0, \beta=2 \mu+1>1$, which is relevant for the generalized Benjamin-Ono equation. The inverse function $\Psi$ is then

$$
\Psi(\eta)=\operatorname{sgn} \eta|\eta|^{\gamma}
$$

with $\gamma=\beta^{-1}$, and the functions $h_{i}$, up to constants and change of variables and an irrelevant phase factor for $h_{2}$, reduce to the form

$$
h(\eta, x)=(2 \pi i)^{-1} \int d \eta^{\prime}\left(\eta^{\prime}-\eta-i 0\right)^{-1}\left|\eta^{\prime} / \eta\right|^{\delta} \exp \left[i x\left(\Psi\left(\eta^{\prime}\right)-\Psi(\eta)\right)\right]
$$

for various values of $\delta$ which depend on $i$. More precisely, from Propositions 2.2 and 2.3 and from (3.5), (3.6) one obtains the relevant values $\delta_{2}=0, \delta_{3}=-1 / 4$ and $\delta_{4}=\gamma / 4-1 / 2$, corresponding to $d \sigma_{2} \cong d \eta, d \sigma_{3} \cong \xi^{\beta / 2-1} d \xi \cong \eta^{-1 / 2} d \eta$ and $d \sigma_{4} \cong \eta^{\gamma / 2-1} d \eta$ respectively. The assumptions of Proposition 2.1 are satisfied by $\Phi$ with $\Omega=\mathbb{R} \backslash\{0\}$, and the limit points $\xi_{*}$ are 0 and $\pm \infty$ with the same exponent $\beta$. By homogeneity, the function $h$ depends only on one variable, namely

$$
h(\eta, x)=h\left(\eta|x|^{\beta}, \pm 1\right) \text { for } x \gtrless 0 .
$$

The basic estimates can then be stated as follows.

Proposition 3.4. Let $0<\gamma<1,-1<\delta<\gamma$ and let $h$ be defined by (3.19), (3.20). Then the following estimates hold for all $\eta \in \mathbb{R}, \eta \neq 0$ :

(1) For $-1<\delta \leq 0$,

$$
|h(\eta, \pm 1)| \leq C \text {. }
$$

For $0 \leq \delta<\gamma$

$$
|h(\eta, \pm 1)| \leq C\left(1+|\eta|^{-\delta}\right) .
$$

(2) For $\gamma-1 \leq \delta<0$,

$$
\left|\eta h^{\prime}(\eta, \pm 1)\right| \leq C_{1}
$$

For $-1<\delta \leq \gamma-1$,

$$
\left|\eta h^{\prime}(\eta, \pm 1)\right| \leq C_{1}\left(1+|\eta|^{\gamma-\delta-1}\right) .
$$

The proof of Proposition 3.4 is given in the Appendix. With that proposition available, it is straightforward to derive the retarded estimates in the case (3.18).

Proposition 3.5. Let $\Phi$ be defined by (3.18) with $\beta>1$. Then

(1) The operator $\left(B_{\sigma_{2}}^{*} B\right)_{R}$ is bounded from $X_{2}$ to $X_{2}^{*}$.

(2) The operator $\left(B_{\tilde{\sigma}_{3}}^{*} B\right)_{R}$ is bounded from $X_{3}$ to $X_{2}^{*}$ and from $X_{2}$ to $X_{3}^{*}$ for $\beta \geq 4 / 3$ (i.e. $\mu \geq 1 / 6$ ). 
(3) The operator $\left(B_{\tilde{\sigma}_{4}}^{*} B\right)_{R}$ is bounded from $X_{4}$ to $X_{2}^{*}$ and from $X_{2}$ to $X_{4}^{*}$ for $\beta \geq 3 / 2$ (i.e. $\mu \geq 1 / 4$ ).

Proof. The results follow immediately from Propositions 3.2, 3.3 and from the estimates (3.21) and (3.23) of Proposition 3.4, which ensure that $h_{2} \in L^{\infty}$ and that $h_{3}$ and $h_{4}$ satisfy the assumptions of Proposition 3.3. The restrictions on $\beta$ in parts (2) and (3) are equivalent to the condition $\gamma-1 \leq \delta_{i}$ for $i=3,4$ respectively, required for (3.23) to hold. QED

Remark 3.2. If $\Phi$ is a polynomial with $\Phi^{\prime}$ non-negative, then the function $h_{2}$ can be computed in a semi-explicity way by the method of residues. In that case $\Phi$ is necessarily of odd degree $2 n+1$, and $d \sigma_{2}(\xi)=\Phi^{\prime}(\xi) d \xi$. For any $\eta \in \mathbb{R}$, the equation $\Phi(\xi)=\eta$ has one real root $\xi_{0}=\xi_{0}(\eta)$ and $n$ pairs of complex conjugate roots $\left(\xi_{j}(\eta), \bar{\xi}_{j}(\eta)\right), 1 \leq j \leq n$, with $\operatorname{Im} \xi_{j}>0$. One then finds from (3.5),

$$
h_{2}(\xi, x)=\theta(x)\left(e^{i x \xi_{0}}+\sum_{1 \leq j \leq n} e^{i x \xi_{J}}\right)-\theta(-x) \sum_{1 \leq j \leq n} e^{i x \bar{\xi}_{J}} .
$$

In particular $\left\|h_{2}\right\|_{\infty}=n+1$ and the boundedness of $\left(B_{\sigma_{2}}^{*} B\right)_{R}$ from $X_{2}$ to $X_{2}^{*}$ follows directly from Proposition 3.2, part (1). That example covers the case of the generalized Korteweg-de Vries equation (3.18) with $\mu=n$ an integer. In that case $\xi_{j}=\xi_{0} \exp [2 \pi i j /(2 n+1)]$.

We now come back to the more general situation (in space dimension 1) covered by Propositions 2.2 and 2.3 and study the boundedness of the retarded operator $\left(B_{\tilde{\sigma}}^{*} B\right)_{R}$ from $X_{3}$ to $X_{4}^{*}$ or equivalently from $X_{4}$ to $X_{3}^{*}$ for the appropriate $\tilde{\sigma}$, namely $\tilde{\sigma}=\left(\sigma_{3} \sigma_{4}\right)^{1 / 2}$ (see Corollary 2.2). For that choice of $\tilde{\sigma}$ and under suitable assumptions on $\Phi$ which are satisfied in particular in the special case (3.18), one can show by an extension of Proportion 2.1 that $\tilde{\sigma}$ satisfies the estimate

$$
\left|\left(\mathscr{F}^{-1} \tilde{\sigma}\right)(t, x)\right| \leq C|t|^{-1 / 4}|x|^{-1 / 4} .
$$

By the HLS inequality, that estimate implies that $\left(B_{\tilde{\sigma}}^{*} B\right)_{R}$ is a bounded operator from $L_{t}^{\bar{q}_{1}}\left(\mathbb{R}, L_{x}^{\bar{r}_{1}}\right)$ to $L_{t}^{q_{2}}\left(\mathbb{R}, L_{x}^{r_{2}}\right)$ and from $L_{x}^{\bar{q}_{1}}\left(\mathbb{R}, L_{t}^{\bar{r}_{1}}\right)$ to $L_{x}^{q_{2}}\left(\mathbb{R}, L_{t}^{r_{2}}\right)$ for $1<q_{i}, r_{i}<\infty$ and $1 / q_{1}+1 / q_{2}=1 / r_{1}+1 / r_{2}=1 / 4$. That result however does not cover the case of $\left(X_{3}, X_{4}^{*}\right)$ or $\left(X_{4}, X_{3}^{*}\right)$ because (i) the case where one of the exponents is 1 or $\infty$ is a forbidden limiting case for the HLS inequality and (ii) more important, an application of that inequality can yield only pairs of spaces where the time and space integrations occur in the same order. Disregarding the difficulty with limiting cases, one would get at best the boundedness of $\left(B_{\tilde{\sigma}}^{*} B\right)_{R}$ from $X_{3}$ to $L_{t}^{\infty}\left(L_{x}^{4}\right)$, which by the Minkowski inequality is larger than $X_{4}^{*}$ and actually strictly so, and similarly from $X_{4}$ to $L_{x}^{\infty}\left(L_{t}^{4}\right)$ which is strictly larger than $X_{3}^{*}$. We shall therefore follow a different track, which consists of artificially restoring the factorisation of Lemma 2.1 for the retarded operator. This can be done at the cost of (i) restricting the time integrals to a bounded interval $I$ and producing constants that tend to blow up as $\log |I|$, and (ii) introducing logarithmic singularities in the time integrals. In the elementary framework of $L^{q}\left(L^{r}\right)$ spaces used so far, logarithms are replaced by small powers, and we end up with the following result.

Proposition 3.6. Let $\Phi$ satisfy the assumptions of Propositions 2.2 and 2.3 and let $\tilde{\sigma}=\left(\sigma_{3} \sigma_{4}\right)^{1 / 2}$. Let $I$ be a bounded interval. Let $2 \leq r<\infty$ and $2 \leq q<4$. Then 
the operator $\left(B_{\tilde{\sigma}}^{*} B\right)_{R}$ is bounded from $L_{t}^{\bar{q}}\left(I, L_{x}^{1}\right)$ to $L_{x}^{4}\left(\mathbb{R}, L_{t}^{r}(I)\right)$ and by duality from $L_{x}^{4 / 3}\left(\mathbb{R}, L_{t}^{\bar{r}}(I)\right)$ to $L_{t}^{q}\left(I, L_{x}^{\infty}\right)$ with

$$
\left\|\left(B_{\tilde{\sigma}}^{*} B\right)_{R} f ; L_{x}^{4}\left(\mathbb{R}, L_{t}^{r}(I)\right)\right\| \leq C a_{q} b_{r}|I|^{1 / r+1 / q-1 / 4}\left\|f ; L_{t}^{\bar{q}}\left(I, L_{x}^{1}\right)\right\|
$$

and a dual estimate with the same constant. The constants $a_{q}$ and $b_{r}$ tend to infinity when $q \rightarrow 4$ and $r \rightarrow \infty$ respectively.

Proof. We use the representation of the $\theta$ function

$$
\theta\left(t-t^{\prime}\right)=\pi^{-1} \int d \tau \theta(t-\tau) \theta\left(\tau-t^{\prime}\right)|t-\tau|^{-1 / 2}\left|\tau-t^{\prime}\right|^{-1 / 2}
$$

to write, for $f, g \in \mathscr{S}_{2}$ with time support in $I$,

$$
\begin{aligned}
\left\langle g,\left(B_{\tilde{\sigma}}^{*} B\right)_{R} f\right\rangle= & \left(4 \pi^{3}\right)^{-1} \int\left(d \sigma_{3}(\xi) d \sigma_{4}(\xi)\right)^{1 / 2} d \tau d t d x d t^{\prime} d x^{\prime} \\
& \times \bar{g}(t, x) \theta(t-\tau)(t-\tau)^{-1 / 2} f\left(t^{\prime}, x^{\prime}\right) \theta\left(\tau-t^{\prime}\right)\left(\tau-t^{\prime}\right)^{-1 / 2} \\
& \times \exp \left[i\left(t-t^{\prime}\right) \eta+i\left(x-x^{\prime}\right) \xi\right],
\end{aligned}
$$

where $\eta=\Phi(\xi)$ and all time integrals, in particular that on $\tau$, are taken in $I$. We now apply the Schwarz inequality in $\xi$ and $\tau$ to obtain

$$
\left|\left\langle g,\left(B_{\tilde{\sigma}}^{*} B\right) f\right\rangle\right|^{2} \leq\left(4 \pi^{3}\right)^{-2} J_{3} J_{4}
$$

with

$$
\begin{aligned}
J_{3}= & \int d t d x d t^{\prime} d x^{\prime} \bar{f}(t, x) f\left(t^{\prime}, x^{\prime}\right) \\
& \times \int d \sigma_{3}(\xi) \exp \left[i\left(t-t^{\prime}\right) \eta+i\left(x-x^{\prime}\right) \xi\right] \\
& \times \int d \tau \theta(\tau-t) \theta\left(\tau-t^{\prime}\right)(\tau-t)^{-1 / 2}\left(\tau-t^{\prime}\right)^{-1 / 2}, \\
J_{4}= & \int d t d x d t^{\prime} d x^{\prime} \bar{g}(t, x) g\left(t^{\prime}, x^{\prime}\right) \\
& \times \int d \sigma_{4}(\xi) \exp \left[i\left(t-t^{\prime}\right) \eta+i\left(x-x^{\prime}\right) \xi\right] \\
& \times \int d \tau \theta(t-\tau) \theta\left(t^{\prime}-\tau\right)(t-\tau)^{-1 / 2}\left(t^{\prime}-\tau\right)^{-1 / 2} .
\end{aligned}
$$

Taking for definiteness $I=[0, T]$ and changing the integration variable from $\tau$ to $t \vee t^{\prime}+\tau\left|t-t^{\prime}\right|$ with $t \vee t^{\prime}=\operatorname{Max}\left(t, t^{\prime}\right)$, we can rewrite the integral over $\tau$ in (3.31) as

$$
\begin{aligned}
\int_{t \vee t^{\prime}}^{T} d \tau(\tau-t)^{-1 / 2}\left(\tau-t^{\prime}\right)^{-1 / 2} & =\int_{0}^{\bar{\tau}} d \tau[\tau(\tau+1)]^{-1 / 2} \\
& =2 \log \left(\bar{\tau}^{1 / 2}+(1+\bar{\tau})^{1 / 2}\right)
\end{aligned}
$$

with $\bar{\tau}=\left|t-t^{\prime}\right|^{-1}\left(T-t \vee t^{\prime}\right)$. That integral is therefore estimated by

$$
\ldots \leq 2 \log 2(1+\bar{\tau})^{1 / 2}=\log 4(1+\bar{\tau}) \leq \log \left(4 T\left|t-t^{\prime}\right|^{-1}\right) .
$$

The same estimate holds for the integral over $\tau$ in (3.32). Using the estimate (2.41) satisfied by $\sigma_{3}$, we can now estimate

$$
\begin{aligned}
J_{3} & \leq C \int d t d x d t^{\prime} d x^{\prime}\left|f(t, x) f\left(t^{\prime}, x^{\prime}\right)\right|\left|t-t^{\prime}\right|^{-1 / 2} \log \left(4 T\left|t-t^{\prime}\right|^{-1}\right) \\
& \leq C\left\|f ; L_{t}^{\bar{q}}\left([0, T], L_{x}^{1}\right)\right\|^{2}\left\||t|^{-1 / 2} \log (4 T / t) ; L^{q / 2}([-T, T])\right\|
\end{aligned}
$$

by the Young inequality,

$$
\ldots=C T^{-1 / 2+2 / q} a_{q}^{2}\left\|f ; L_{t}^{\bar{q}}\left(I, L_{x}^{1}\right)\right\|^{2}
$$


with

$$
a_{q}^{2}=\left\||t|^{-1 / 2} \log (4 / t) ; L^{q / 2}([-1,1])\right\|
$$

by homogeneity. Similarly we use the estimate (2.45) satisfied by $\sigma_{4}$ to estimate

$$
\begin{aligned}
J_{4} & \leq C \| g ; L_{x}^{4 / 3}\left(\mathbb{R}, L_{t}^{\bar{r}}([0, T])\left\|^{2}\right\| \log (4 T / t) ; L^{r / 2}([-T, T]) \|\right. \\
& =C T^{2 / r} b_{r}^{2}\left\|g ; L_{x}^{4 / 3}\left(\mathbb{R}, L_{t}^{\bar{r}}(I)\right)\right\|^{2}
\end{aligned}
$$

with

$$
b_{r}^{2}=\left\|\log (4 / t) ; L^{r / 2}([-1,1])\right\| .
$$

The boundedness properties of $\left(B_{\tilde{\sigma}}^{*} B\right)_{R}$ and the estimates (3.27) and its dual follow immediately from (3.30), (3.34), and (3.35). QED

In conclusion, the estimates of $B_{\tilde{\sigma} .}^{*} B$ between the spaces $X_{i}$ and $X_{j}^{*}$ corresponding to the extremal cases of Propositions 2.2 and 2.3 extend to the retarded operators $\left(B_{\tilde{\sigma} .}^{*} B\right)_{R}$ with the following qualifications:

(1) in a straightforward way if $i=1$ or $j=1$ or $(i, j)=(3,3)$ or $(4,4)$.

(2) in finite time intervals and with a small loss of regularity in time if $(i, j)=(3,4)$ or $(4,3)$ (Proposition 3.6).

(3) In the special case (3.18) if $i=2$ or $j=2,2 \leq i, j \leq 4$, under the conditions of Proposition 3.5.

The last result certainly extends to a level of generality comparable to that of Propositions 2.2 and 2.3, with one restriction to be discussed in Remark 3.3 below.

Remark 3.3. Most of the results of Propositions 3.2-3.6 extend to the case where $\Phi$ is not monotonous from $\mathbb{R}$ to $\mathbb{R}$, in the line of Remark 2.1 , with one exception that will be mentioned below. Proposition 3.2 still holds with the functions $h_{2}, h_{i}$ and $K_{i}(i=3,4)$ now being finite sums of expressions of the type (3.5), (3.6), (3.7) corresponding to the intervals of monotonicity of $\Phi$. Proposition 3.3 is insensitive to that generalisation and Proposition 3.6 extends in a straightforward way to the more general situation. At the level of generality of Proposition 3.4, the most natural and interesting example of a non-monotonous $\Phi$ is $\Phi(\xi)=|\xi|^{2 \mu}$. In that case, the estimates of Proposition 3.4 still hold for $\delta \neq 0$, so that Parts (2) and (3) of Proposition 3.5 still hold in that case (now with $\gamma^{-1}=\beta=2 \mu$ ). On the other hand, for $\Phi$ even, $\Phi$ a bijection from $\mathbb{R}^{+}$to $\mathbb{R}^{+}$with inverse map $\Psi$, the function $h_{2}$ that occurs in (3.8) is now replaced by

$$
h_{2+}(\eta, x)=(i \pi)^{-1} \int_{0}^{\infty} d \eta^{\prime}\left(\eta^{\prime}-\eta-i 0\right)^{-1} \cos \left[x \Psi\left(\eta^{\prime}\right)\right]
$$

and for $\Phi(\xi)=|\xi|^{2 \mu}$ (and in more general circumstances), that function develops a logarithmic singularity for small $\eta$ (or small $x$ ) (see Remark A.1) so that Part (1) of Proposition 3.5 does not hold in that case. This fact can be remedied by considering instead of $\left(B_{\sigma_{2}}^{*} B\right)_{R}$ the operator $H\left(B_{\sigma_{2}}^{*} B\right)_{R}$, where $H$ is the Hilbert transform in $x$, or equivalently multiplication by $i$ sgn $\xi$ in Fourier transformed variables. With that replacement in (3.8), $h_{2+}$ is replaced by $h_{2-}$ defined by

$$
h_{2-}(\eta, x)=i \pi^{-1} \int_{0}^{\infty} d \eta^{\prime}\left(\eta^{\prime}-\eta-i 0\right) \sin \left[x \Psi\left(\eta^{\prime}\right)\right]
$$


and $h_{2-} \in L^{\infty}$ for $\Phi(\xi)=|\xi|^{2 \mu}$, so that $H\left(B_{\sigma_{2}}^{*} B\right)_{R}$ is bounded from $X_{2}$ to $X_{2}^{*}$. In the special case where $\Phi$ is an even polynomial with $\xi \Phi^{\prime}(\xi) \geq 0, h_{2}$ can actually be computed by the method of residues in the same way as in Remark 3.2. For instance, in the case $\Phi(\xi)=\xi^{2}$ of the Schrödinger equation, one finds

$$
\begin{aligned}
h_{2-} & =(2 \pi)^{-1} \int 2 \xi^{\prime} d \xi^{\prime}\left(\xi^{\prime 2}-\eta-i 0\right)^{-1} \exp \left(i x \xi^{\prime}\right) \\
& =i \theta(x) \exp \left[i x \eta^{1 / 2}\right]-i \theta(-x) \exp \left[-i x \eta^{1 / 2}\right]
\end{aligned}
$$

with $\eta^{1 / 2}=|\eta|^{1 / 2}$ if $\eta>0$ and $\eta^{1 / 2}=i|\eta|^{1 / 2}$ if $\eta<0$.

We conclude this section by describing briefly an application of the previous results to the Cauchy problem for the generalized Benjamin-Ono equation

$$
\partial_{t} u-L u=D V^{\prime}(u)
$$

where $u$ is a real function defined in space time $\mathbb{R}^{2}, \partial_{t}=\partial / \partial t, D=\partial / \partial x$, $L=i \Phi(-i D)$ (cf. (2.25)) with $\Phi$ defined by (3.18), or equivalently $L=D|D|^{2 \mu}$, and $V \in \mathscr{C}^{1}(\mathbb{R}, \mathbb{R})$ with $V(0)=V^{\prime}(0)=0$. That equation reduces to the usual Korteweg-de Vries equation for $\mu=1$ and to the usual Benjamin-Ono equation for $\mu=1 / 2$ in the special case $V^{\prime}(u)=u^{2}$. The Cauchy problem for that equation with initial data $u(0)=u_{0}$ is equivalent to the equation

$$
u=A^{*} u_{0}+\left(A^{*} A\right)_{R} \theta(t) D V^{\prime}(u)
$$

with $A, A^{*}$ defined by (2.5), (2.6) and $U(t)=\exp (t L)$ as before.

A large amount of work has been devoted to that problem, especially in the special cases $\mu=1$ and $\mu=1 / 2$ (see [1, 10, 13, 17] and references therein quoted). In the present framework, as explained in Sect. 2 [see especially (2.31)], one is led to treat that problem as follows: one looks for admissible pairs $(\sigma, X)$ associated with $\Sigma=\{\xi, \eta=\Phi(\xi)\}$, one takes initial data $u_{0} \in \mathscr{H}=\mathscr{F}{ }^{-1} L^{2}(\Sigma, \sigma)$ for such a $\sigma$, and one tries to solve (3.40) by contraction in $W^{-1} X^{*}$ or in an intersection of such spaces. For that purpose, one has to ensure that $\left(A^{*} A\right)_{R}$ is bounded from $X$ to $W^{-1} X^{*}$, or equivalently that $\left(B_{\sigma}^{*} B\right)_{R}$ is bounded from $X$ to $X^{*}$, and that the map $u \rightarrow D V^{\prime}(u)$ is bounded from $W^{-1} X^{*}$ to $X$. The main difficulty with that scheme comes from the derivative in $D V^{\prime}(u)$, which has to be compensated by the smoothing properties of $\left(A^{*} A\right)_{R}$. Now it follows from (2.31) that if $(\sigma, X)$ is an admissible pair with $X$ some space such as occur in Propositions 2.2 and 2.3 and if $d \sigma(\xi)=w(\xi) d \xi$, then the smoothing expected from $\left(A^{*} A\right)_{R}$ is $w(-i D)$. More generally, the smoothing from $X_{i}$ to $X_{j}^{*}$ for two such admissible pairs $\left(\sigma_{i}, X_{i}\right)$ with $d \sigma_{i}=w_{i} d \xi$ is $\left[w_{i}(-i D) w_{j}(-i D)\right]^{1 / 2}$. We have at our disposal the spaces $X$ of Propositions 2.2 and 2.3, and we continue to label the extreme cases thereof as $X_{i}, 1 \leq i \leq 4$, in keeping with the notation of this section. With $\Phi$ given by (3.18), the associated measures $\sigma_{i}$ become $d \sigma_{1}(\xi)=d \xi$, $d \sigma_{2}(\xi)=|\xi|^{2 \mu} d \xi, d \sigma_{3}(\xi)=|\xi|^{\mu-1 / 2} d \xi$ and $d \sigma_{4}(\xi)=\xi^{-1 / 2} d \xi$, and the maximum amount of smoothing, namely $2 \mu$, is associated with the space $X_{2}$. If one uses the boundedness of the retarded operator in the simple case where one of the spaces is $X_{1}$, then the maximal amount of smoothing is $\mu$, corresponding to the pair $\left(X_{1}, X_{2}^{*}\right)$ and one can set up a contraction scheme for $\mu \geq 1$ only [17]. On the other hand, if one uses the pair $\left(X_{2}, X_{2}^{*}\right)$, and for that purpose, Proposition 3.5 , the amount of smoothing is $2 \mu$, thereby allowing for a contraction scheme to work down to $\mu=1 / 2$. 
Following this path, one can prove the following result, which we state in a somewhat loose way in order to avoid technicalities. We use the standard Sobolev space $H^{s}$ defined by the norm

$$
\left\|v ; H^{s}\right\|^{2}=\int d \xi\left(1+\xi^{2}\right)^{s}|\hat{v}(\xi)|^{2} .
$$

Let $\mu>1 / 2,0 \leq s \leq 1 / 2,2 s>1-\mu$, and let $V \in \mathscr{C}^{2}(\mathbb{R}, \mathbb{R})$ satisfy

$$
\left|V^{\prime \prime}\left(\varrho_{1}\right)-V^{\prime \prime}\left(\varrho_{2}\right)\right| \leq C\left|\varrho_{1}-\varrho_{2}\right| \sum_{i, j=1,2}\left|\varrho_{i}\right|^{p_{J}-1}
$$

for some $p_{1}, p_{2}$ satisfying

$$
\operatorname{Max}\left\{\frac{2(\mu+1)}{\mu+s}, \frac{2(\mu+1)}{\mu+1 / 4}\right\}<p_{1} \leq p_{2}<\frac{4 \mu}{1-2 s}
$$

and all $\varrho_{1}, \varrho_{2}$ in $\mathbb{R}$. Let $u_{0} \in H^{s}$. Then the Cauchy problem for (3.39) can be solved locally in time by a contraction method; namely there exists $T>0$ depending on $\left\|u_{0} ; H^{s}\right\|$ such that (3.40) has a unique solution

$$
u \in \mathscr{C}\left(I, H^{s}\right) \cap \mathscr{X}(I) .
$$

The space $\mathscr{X}(I)$ is an intersection of spaces of the type $X_{2}^{*}$ and $X_{4}^{*}$ at the level of regularity naturally associated with the fact that $u_{0} \in H^{s}$, and with the additional complication that Sobolev spaces have to be replaced by Besov spaces in its definition, in order to cover the case of non-linearities as general as allowed by (3.41).

One sees in particular that the value $p=4$ is always allowed by the condition (3.42) and that for $p=4$ one can solve the Cauchy problem for (3.39) with initial data $u_{0}=H^{s}$ for $2 s>1-\mu$, and in particular for $s$ arbitrarily small if $\mu=1$. The upper bound in (3.42) is optimal, but we have made no effort to optimize the lower bound, and that given in (3.42) is not significant.

The details will be given elsewhere.

\section{Appendix}

This appendix is devoted to the proofs of Propositions 2.1, 3.3, and 3.4. Those proofs are based on standard stationary phase estimates. That of Proposition 2.1 is a minor variation of the proof given in [15]. We begin with two elementary lemmas.

Lemma A.1. Let $I=[a, b], \Phi \in \mathscr{C}^{2}(I, \mathbb{R})$ with $\Phi^{\prime}>0, \Phi^{\prime \prime}>0$ and $\varphi \in \mathscr{C}(I, \mathbb{R})$ with bounded variation. Then

$$
\left|\int_{a}^{b} \varphi e^{i \Phi}\right| \leq 2 \Phi^{\prime}(a)^{-1}\left(|\varphi(a)|+\int_{a}^{b}|d \varphi|\right) .
$$

Proof. Integration by parts yields

$$
\int_{a}^{b} \varphi e^{i \Phi}=\left.e^{i \Phi}\left(i \Phi^{\prime}\right)^{-1} \varphi\right|_{a} ^{b}+i \int_{a}^{b} e^{i \Phi} d\left(\varphi / \Phi^{\prime}\right)
$$


so that

$$
\begin{aligned}
|\cdot| & \leq|\varphi(a)| \Phi^{\prime}(a)^{-1}+|\varphi(b)| \Phi^{\prime}(b)^{-1}+\int_{a}^{b}\left|d\left(\varphi / \Phi^{\prime}\right)\right| \\
& \leq 2|\varphi(a)| \Phi^{\prime}(a)^{-1}+\int_{a}^{b}\left(\left|d\left(\varphi / \Phi^{\prime}\right)\right|+d\left(|\varphi| / \Phi^{\prime}\right)\right) \\
& \leq 2|\varphi(a)| \Phi^{\prime}(a)^{-1}+2 \int_{a}^{b} \Phi^{\prime-1}|d \varphi|
\end{aligned}
$$

since $\Phi^{\prime}$ is increasing. (A.1) follows immediately from (A.2). QED

Lemma A.2. Let $I \subset \mathbb{R}$ be an interval, $\Phi \in \mathscr{C}^{2}(I, \mathbb{R})$ with $\Phi^{\prime \prime} \geq m>0$ and $\Phi^{\prime \prime}$ of bounded variation in $I$, namely

$$
v=\int_{I}\left|d \Phi^{\prime \prime}\right|<\infty
$$

Then

$$
\left|\int_{I} \Phi^{\prime 1 / 2} e^{i \Phi}\right| \leq 4(2+v / m)^{1 / 2}
$$

Proof. Let $I=(a, b)$ ( $a$ and/or $b$ can be infinite). There is at worst one point of stationary phase in $I$, which we take to be zero. We consider the intervals $(a, 0]$ and $[0, b)$ separately and add their contributions. Let $0<\varepsilon \leq b$. We estimate

$$
\left|\int_{0}^{b} \Phi^{\prime \prime 1 / 2} e^{i \Phi}\right| \leq \int_{0}^{\varepsilon} \Phi^{\prime 1 / 2}+2 \Phi^{\prime}(\varepsilon)^{-1}\left(\Phi^{\prime \prime}(\varepsilon)^{1 / 2}+\int_{a}^{b}\left|d \Phi^{\prime 1 / 2}\right|\right)
$$

by Lemma A.1,

$$
\ldots \leq m^{-1 / 2} \Phi^{\prime}(\varepsilon)+2 \Phi^{\prime}(\varepsilon)^{-1} M
$$

with

$$
M=\Phi^{\prime \prime}(\varepsilon)^{1 / 2}+\int_{\varepsilon}^{b}\left|d \Phi^{\prime \prime 1 / 2}\right| \leq m^{1 / 2}\left(1+v_{+} / m\right)
$$

and $v_{+}=\int_{0}^{b}\left|d \Phi^{\prime \prime}\right|$. We optimise in $\varepsilon$ by taking

$$
\Phi^{\prime}(\varepsilon)^{2}=2 M m^{1 / 2}=2\left(m+v_{+}\right) .
$$

(If $\Phi^{\prime}(b)<2 M m^{1 / 2}$, we take simply $\varepsilon=b$.) Then

$$
\left|\int_{0}^{b} \Phi^{\prime \prime 1 / 2} e^{i \Phi}\right| \leq 2 \sqrt{2}\left(1+v_{+} / m\right)^{1 / 2}
$$

from which (A.3) follows by adding the contributions of $(a, 0]$ and $[0, b)$. QED Proof of Proposition 2.1. We first extract from $\Omega$ a finite number of compact intervals, the contribution of which is correctly estimated by Lemma A.2, so as to be left with sufficiently small neighborhoods of points of $\partial \Omega$. After suitable 
changes of variables and of signs, the latter can be reduced to a finite number of intervals of the type $[0,1]$ or $[1, \infty)$, where $\Phi_{0}$ satisfies $\Phi_{0}^{\prime \prime}>0$ and $(2.34),(2.35)$. We treat those two cases together by considering the interval $[0, \infty)$ with those two conditions. That interval contains at worst one stationary phase point $\xi_{0}$. We separate out the contribution of the interval $\left[\xi_{0} / 2,2 \xi_{0}\right]$ which we estimate by Lemma A.2 as

$$
\left|\int_{\xi_{0} / 2}^{2 \xi_{0}} \Phi_{0}^{\prime \prime 1 / 2} e_{0}^{i \Phi}\right| \leq 4|t|^{-1 / 2}(2+v / m)^{1 / 2} \leq C|t|^{-1 / 2}
$$

since

$$
\begin{gathered}
m=\operatorname{Inf}_{\xi_{0} / 2 \leq \xi \leq 2 \xi_{0}} \Phi_{0}^{\prime \prime}(\xi) \geq c_{1} 2^{-|\beta-2|} \xi_{0}^{\beta-2} \\
v=\int_{\xi_{0} / 2}^{\xi_{0}}\left|d \Phi_{0}^{\prime \prime}\right| \leq c_{3}\left(1+2^{2-\beta}\right) \xi_{0}^{\beta-2} .
\end{gathered}
$$

For $\xi \notin\left[\xi_{0} / 2,2 \xi_{0}\right], \Phi_{0}^{\prime}$ stays away from zero. For instance for $\xi \geq 2 \xi_{0}$,

$$
\Phi_{0}^{\prime}(\xi) \geq \int_{\xi / 2}^{\xi} \Phi_{0}^{\prime \prime} \geq c_{1}(\beta-1)^{-1}\left(1-2^{1-\beta}\right) \xi^{\beta-1} \geq C \xi^{\beta-1},
$$

and similarly for $\xi \leq \xi_{0} / 2$. We assume for definiteness that $\beta>0$ and we split $\mathbb{R}_{+}$as

$$
\begin{gathered}
\mathbb{R}_{+}=\bigcup_{j=0}^{\infty} I_{j}, \\
I_{0}=\left[0, \xi_{1}\right] \quad \text { with } \xi_{1}=|t|^{-1 / \beta}, \\
I_{j}=\left[2^{j-1} \xi_{1}, 2^{j} \xi_{1}\right] \text { for } j \geq 1 .
\end{gathered}
$$

We estimate the contribution of $I_{0}$ by

$$
\left|\int_{I_{0}} \Phi_{0}^{\prime \prime 1 / 2} e^{i t \Phi_{0}}\right| \leq \int_{I_{0}} \Phi_{0}^{\prime 1 / 2} \leq 2 c_{2}^{1 / 2} \beta^{-1}|t|^{-1 / 2},
$$

and we estimate the contribution of that part of $I_{j}$ which is not contained in $\left[\xi_{0} / 2,2 \xi_{0}\right]$ by the use of Lemma A.1, of (A.5) and (2.34), (2.35), thereby obtaining

$$
\left|\int_{I_{J}} \Phi_{0}^{\prime \prime 1 / 2} e^{i t \Phi_{0}}\right| \leq C|t|^{-1}\left(2^{j} \xi_{1}\right)^{1-\beta}\left(2^{j} \xi_{1}\right)^{\beta / 2-1}=C|t|^{-1 / 2} 2^{-j \beta / 2} .
$$

Summing (A.7) over $j$ and adding (A.4) and (A.6), we obtain (2.36). For $\beta<0$, we use the same method, except that the $L^{1}$ estimate is now used in the interval $\left[\xi_{1}, \infty\right)$ instead of $\left[0, \xi_{1}\right]$. It is obvious from the proof that the constant $C_{\Phi_{0}}$ depends only on $\Phi_{0}^{\prime \prime}$. QED

Proof of Proposition 3.3. For any function $f$ of bounded variation in $I=[a, b]$, we use the notation

$$
\operatorname{Var} f(I)=\int_{I}|d f|, \quad|\partial| f(I)=|f(a)|+|f(b)| .
$$


The proof of Proposition 3.3 is a variation of that of Proposition 2.1. It consists in decomposing $\Omega$ as a union of intervals in the same way as in the latter, and adding the contribution of those intervals. In each such interval, we use one of the following two estimates.

(i) an $L^{1}$ estimate. In that case, inclusion of $h$ yields the same estimate multiplied by $\|h\|_{\infty}$.

(ii) an estimate of the type $(\operatorname{Var}+|\partial|) f$ with $f=\left|\Phi_{0}^{\prime}\right|^{-1}\left|\Phi_{0}^{\prime \prime}\right|^{1 / 2}$.

Inclusion of $h$ replaces such an estimate by

$$
\begin{aligned}
(\operatorname{Var}+|\partial|)(f h) & \leq\|h\|_{\infty}(\operatorname{Var}+|\partial|) f+(\operatorname{Var} h)\|f\|_{\infty} \\
& \leq\left(\|h\|_{\infty}+\operatorname{Var} h\right)(\operatorname{Var}+|\partial|) f .
\end{aligned}
$$

It is therefore sufficient to prove that $\operatorname{Var} h(I)$ is uniformly bounded in $I$ for those intervals $I$ that occur in the proof of Proposition 2.1 and for which the second type of estimate is used. In a compact $K \subset \Omega$ excluding sufficiently small neighborhoods of points of $\partial \Omega$, we estimate

$$
\operatorname{Var} h(K) \leq\left\|h^{\prime} ; L^{1}(K)\right\| .
$$

In the remaining neighborhoods of points $\xi_{*} \in \partial \Omega$, which we take as before to be either $\xi_{*}=0$ or $\xi_{*}=\infty$, the relevant intervals are of the type $I=[\xi, a \xi]$ for some fixed $a>1$ and variable $\xi$. In such an interval

$$
\operatorname{Var} h \leq \int_{\xi}^{a \xi} \xi^{\prime-1} d \xi^{\prime}\left\|\xi^{\prime} d h / d \xi^{\prime}\right\|_{\infty} \leq\|\xi d h / d \xi\|_{\infty} \log a,
$$

and the result follows from (3.15), (3.16). QED

Proof of Proposition 3.4. By Remark 3.1, one can replace the $i 0$ prescription by a principal value prescription in the integral over $\eta^{\prime}$. Furthermore, it is sufficient to consider the plus sign in $h$ and the case $\eta>0$.

Part (1). For any interval $I$, we define

$$
g(\eta, I)=\int_{I} d \eta^{\prime}\left|\eta^{\prime}\right|^{\delta}\left(\eta^{\prime}-\eta\right)^{-1} \exp \left[i \Psi\left(\eta^{\prime}\right)\right],
$$

where the integral is a principal value if $\eta \in I$. We use three types of estimates.

Estimate 1. For $2 a \leq \eta$, we rewrite

$$
\begin{aligned}
g(\eta,[\eta-a, \eta+a])= & \int_{0}^{a} \zeta^{-1} d \zeta\left\{|\eta+\zeta|^{\delta} \exp [i \Psi(\eta+\zeta)]\right. \\
& \left.-|\eta-\zeta|^{\delta} \exp [i \Psi(\eta+\zeta)]\right\},
\end{aligned}
$$

and we estimate

$$
\begin{aligned}
& |g(\eta,[\eta-a, \eta+a])| \\
& \quad \leq \int_{0}^{a} \zeta^{-1} d \zeta\left\{\left|(\eta+\zeta)^{\delta}-(\eta-\zeta)^{\delta}\right|+\eta^{\delta}(\Psi(\eta+\zeta)-\Psi(\eta-\zeta))\right\} \\
& \quad \leq C a\left(\eta^{\delta-1}+\eta^{\delta+\gamma-1}\right) \leq C \eta^{\delta}
\end{aligned}
$$

if either $2 a=\eta \leq 1$ or $2 a=\eta^{1-\gamma} \geq 1$. 
Estimate 2. For any compact interval $I$ not containing 1, we estimate

$$
|g(\eta, \eta I)| \leq \eta^{\delta} \int_{I} d \eta^{\prime}\left|\eta^{\prime}\right|^{\delta}\left|\eta^{\prime}-1\right|^{-1}=C \eta^{\delta} .
$$

Estimate 3. By integration by parts, for any closed interval $I$ not containing 0 or $\eta$, we estimate

$$
|g(\eta, I)| \leq(\operatorname{Var}+|\partial|) f(I) \leq 4\left\|f ; L^{\infty}(I)\right\|
$$

where

$$
f\left(\eta^{\prime}\right)=\left|\eta^{\prime}\right|^{\delta}\left|\eta^{\prime}-\eta\right|^{-1} \Psi^{\prime}\left(\eta^{\prime}\right)^{-1}=\gamma^{-1}\left|\eta^{\prime}\right|^{\delta+1-\gamma}\left|\eta^{\prime}-\eta\right|^{-1}
$$

since $f$ has at most one extremum in $I$, at $\eta^{\prime}=\eta_{m} \equiv-\eta(\gamma-\delta)(1+\delta-\gamma)^{-1}<\eta / 2$.

We now estimate $g(\eta, \mathbb{R})$ separately for $\eta \geq 1$ and $\eta \leq 1$.

For $\eta \geq 1$, we use

- the estimate (A.13) in $[\eta-a, \eta+a]$ with $2 a=\eta^{1-\gamma} \geq 1$,

- the estimate (A.14) with $I=[-1,1 / 2]$ in the interval $[-\eta, \eta / 2]$,

- the estimate (A.15) in the remaining intervals $(-\infty,-\eta],[\eta / 2, \eta-a]$ and $[\eta+a,+\infty)$.

In all cases, we obtain estimates of the form $C \eta^{\delta}$. Note in particular that $a$ has been chosen so that $f(\eta \pm a)=C \eta \delta$. Collecting those estimates yields (3.21) for $\eta \geq 1$, independently of the sign of $\delta$.

For $\eta \leq 1$, we use

- the estimate (A.13) in $[\eta / 2,3 \eta / 2]$, thereby obtaining $C \eta^{\delta}$,

- the estimate (A.14) in $[-\eta, \eta / 2]$ and $[3 \eta / 2,3 \eta]$ thereby obtaining again $C \eta^{\delta}$,

- the estimate (A.15) in $(-\infty, \eta-2]$ and $[\eta+2, \infty)$ thereby obtaining a constant (which is not as good as $\eta^{\delta}$ for $\delta>0$ ).

In the remaining intervals $[\eta-2,-\eta]$ and $[3 \eta, \eta+2]$, we could use a rough $L^{1}$ estimate for $\delta \neq 0$, namely

$$
\begin{aligned}
|g(\eta,[\eta-2,-\eta] \cup[3 \eta, \eta+2])| & \leq \int_{2 \eta}^{2} \zeta^{-1} d \zeta\left[(\zeta+\eta)^{\delta}+(\zeta-\eta)^{\delta}\right] \\
& \leq 4 \int_{2 \eta}^{2} \zeta^{-1} d \zeta \zeta^{\delta}=8|\delta|^{-1}\left|1-\eta^{\delta}\right|
\end{aligned}
$$

However for $\delta=0$, we need to take advantage of the principal value cancellation. More generally for any $\delta$ we estimate

$$
\begin{aligned}
& \mid g(\eta,[\eta-2,-\eta] \cup[3 \eta, \eta+2]) \\
& \quad \leq \int_{2 \eta}^{2} \zeta^{-1} d \zeta\left\{(\zeta+\eta)^{\delta}-(\zeta-\eta)^{\delta}+\zeta^{\delta}(\Psi(\eta+\zeta)-\Psi(\eta-\zeta))\right\} \\
& \quad \leq \int_{2 \eta}^{2} \zeta^{-1} d \zeta\left(4|\delta| \eta \zeta^{\delta-1}+2 \zeta^{\gamma+\delta}\right) \\
& \quad \leq 2^{1+\delta}\left|1-\eta^{\delta}\right|+2^{1+\gamma+\delta} \gamma^{-1} \operatorname{Max}\left(1, \eta^{\delta}\right) \\
& \quad \leq 4\left(1+2 \gamma^{-1}\right) \operatorname{Max}\left(1, \eta^{\delta}\right) .
\end{aligned}
$$

Collecting the previous estimates yields (3.21), (3.22) for $\eta \leq 1$. 
Part (2). With $\xi=\Psi(\eta)=\operatorname{sgn} \eta|\eta|^{\gamma}$ and similarly $\xi^{\prime}=\Psi\left(\eta^{\prime}\right)$, we compute

$$
\begin{aligned}
& (\eta d / d \eta) \int d \eta^{\prime}\left|\eta^{\prime} / \eta\right|^{\delta}\left(\eta^{\prime}-\eta\right)^{-1} \exp \left[i\left(\xi^{\prime}-\xi\right)\right] \\
& \quad=\gamma(\xi d / d \xi) \int d \eta^{\prime}\left|\eta^{\prime}\right|^{\delta}\left(\eta^{\prime}-1\right)^{-1} \exp \left[i \xi\left(\xi^{\prime}-1\right)\right] \\
& \quad=i \gamma \xi \int d \eta^{\prime}\left|\eta^{\prime}\right|^{\delta}\left(\xi^{\prime}-1\right)\left(\eta^{\prime}-1\right)^{-1} \exp \left[i \xi\left(\xi^{\prime}-1\right)\right] \\
& \quad=i \gamma \int d \eta^{\prime}\left|\eta^{\prime} / \eta\right|^{\delta}\left(\xi^{\prime}-\xi\right)\left(\eta^{\prime}-\eta\right)^{-1} \exp \left[i\left(\xi^{\prime}-\xi\right)\right]
\end{aligned}
$$

by changing the integration variable from $\eta^{\prime}$ to $\eta \eta^{\prime}$ and from $\eta^{\prime}$ to $\eta^{\prime} / \eta$ before and after taking the derivative. Note that the singularity at $\eta^{\prime}=\eta$ has now disappeared. The integrability at $\eta^{\prime}=0$ is ensured as before by the condition $\delta>-1$. For any interval $I$, we define

$$
g_{1}(\eta, I)=i \gamma \int d \eta^{\prime}\left|\eta^{\prime} / \eta\right|^{\delta}\left(\xi^{\prime}-\xi\right)\left(\eta^{\prime}-\eta\right)^{-1} \exp \left[i\left(\xi^{\prime}-\xi\right)\right]
$$

so that the last member of (A.19) if $g_{1}(\eta, \mathbb{R})$. We estimate $g_{1}$ by a combination of $L^{1}$ estimates and integration by parts estimates. The latter yield

$$
\left|g_{1}(\eta, I)\right| \leq(\operatorname{Var}+|\partial|) f_{\eta}(I),
$$

where

$$
f_{\eta}\left(\eta^{\prime}\right)=\left|\eta^{\prime} / \eta\right|^{\delta}\left|\xi^{\prime}-\xi\right|\left|\eta^{\prime}-\eta\right|^{-1}\left|\eta^{\prime}\right|^{1-\gamma}=f_{1}\left(\eta^{\prime} / \eta\right)
$$

by homogeneity, so that for fixed $I$

$$
(\operatorname{Var}+|\partial|) f_{\eta}(\eta I)=(\operatorname{Var}+|\partial|) f_{1}(I)
$$

independently of $\eta$. Furthermore, the function $f_{\eta}$ is piecewise monotonous for a finite decomposition of $\mathbb{R}$ in intervals.

If $\delta \geq \gamma-1, f_{\eta}$ is bounded, so that by (A.20) and (A.22) with $I=\mathbb{R}$,

$$
\left|g_{1}(\eta, \mathbb{R})\right| \leq(\operatorname{Var}+|\partial|) f_{1}(\mathbb{R})=C_{1}<\infty,
$$

which proves (3.23).

If $\delta<\gamma-1$, we consider the cases $\eta \leq 1$ and $\eta \geq 1$ separately.

For $\eta \leq 1$, we use the $L^{1}$ estimate for $\left|\eta^{\prime}\right| \leq \eta$ and obtain

$$
\left|g_{1}(\eta,[-\eta, \eta])\right| \leq C \eta^{\gamma}
$$

by homogeneity, and the estimate (A.20) for $\left|\eta^{\prime}\right| \geq \eta$, thereby obtained

$$
\left|g_{1}(\eta, \mathbb{R} \backslash[-\eta, \eta])\right| \leq(\operatorname{Var}+|\partial|) f_{1}(\mathbb{R} \backslash[-1,1])=C .
$$

For $\eta \geq 1$, we use the $L^{1}$ estimate for $\left|\eta^{\prime}\right| \leq 1$, thereby obtaining

$$
\left|g_{1}(\eta,[-1,1])\right| \leq\left\|\left(\xi^{\prime}-1\right)\left(\eta^{\prime}-1\right)^{-1}\right\|_{\infty}(\delta+1)^{-1} \eta^{\gamma-\delta-1}
$$

and the estimate (A.20) for $\left|\eta^{\prime}\right| \geq 1$, thereby obtaining

$$
\begin{aligned}
\left|g_{1}(\eta, \mathbb{R} \backslash[-1,1])\right| & \leq(\operatorname{Var}+|\partial|) f_{\eta}(\mathbb{R} \backslash[-1,1]) \\
& \leq 2\left(f_{\eta}(1)+f_{\eta}(-1)\right)+C \\
& \leq C\left(1+\eta^{\gamma-\delta-1}\right) .
\end{aligned}
$$

Adding (A.24), (A.25) for $\eta \leq 1$, and (A.26), (A.27) for $\eta \geq 1$ yields (3.24). QED Remark A.1. If $\delta=0$, the cancellation contained in (A.18) is essential, and the contribution of $I=\mathbb{R}^{+}$in (A.12) exhibits a logarithmic singularity in $\eta$ for small $\eta$. In fact, for $\delta=0$ and $\eta \leq 1$, the contributions of the intervals $[0,2 \eta]$ and 
$[a, \infty)$ for fixed finite $a$ are adequately controlled by the previous estimates. Let now $\Psi(a)=\pi / 3$. Then

$$
\operatorname{Re} g(\eta,[2 \eta, a])=\int_{2 \eta}^{a} d \eta^{\prime}\left(\eta^{\prime}-\eta\right)^{-1} \cos \left(\Psi\left(\eta^{\prime}\right)\right) \geq(1 / 2) \log \left[(a-\eta) \eta^{-1}\right] .
$$

Similarly, the function $h_{2+}(\eta, x)$ considered in Remark 3.3 above exhibits a logarithmic singularity for small $\eta$ (or small $x$ ). On the other hand the function $h_{2-}(\eta, x)$ is clearly bounded.

\section{References}

1. Abdelouhad, L., Bona, J.L., Felland, M., Saut, J.C.: Non-local models for non-linear dispersive waves. Physica D 40, 360-392 (1989)

2. Bergh, J., Löfström, J.: Interpolation spaces. Berlin, Heidelberg, New York: Springer 1976

3. Brenner, P.: On $L^{p}-L^{p \prime}$ estimates for the wave equation. Math. Z. 145, 251-254 (1975)

4. Brenner, P.: On scattering and everywhere defined scattering operators for non-linear KleinGordon equations. J. Diff. Eq. 56, 310-344 (1985)

5. Constantin, P., Saut, J.C.: Local smoothing properties of dispersive equations. J. Am. Math. Soc. 1, 413-439 (1988)

6. Ginibre, J.: Le problème de Cauchy pour les équations de Korteweg-de Vries et de Benjamin-Ono généralisées. Publ. IRMAR, Univ. Rennes (1991)

7. Scattering theory in the energy space for a class of non-linear Schrödinger equations. J. Math. Pure Appl. 64, 363-401 (1985)

8. Ginibre, J., Velo, G.: The global Cauchy problem for the non-linear Klein-Gordon equation. Math. Z. 189, 487-505 (1985)

9. Ginibre, J., Velo, G.: Time decay of finite energy solutions of the non-linear Klein-Gordon and Schrödinger equations. Ann. IHP (Phys. Théor.) 43, 399-442 (1985)

10. Ginibre, J., Velo, G.: Smoothing properties and existence of solutions for the generalized Benjamin-Ono equation. J. Diff. Eq. 93, 150-212 (1991)

11. Hörmander, L.: The analysis of linear partial differential operators. I. Berlin, Heidelberg, New York: Springer 1983

12. Kato, T.: Wave operators and similarity for some non-self adjoint operators. Math. Ann. 162, 258-279 (1966)

13. Kato. T.: On the Cauchy problem for the (generalized) Korteweg-de Vries equation. Studies in Applied Math. Adv. Math. Suppl. Studies 18, 93-128 (1983)

14. Kato, T.: On non-linear Schrödinger equations. Ann IHP (Phys. Théor.) 46, 113-129 (1987)

15. Kenig, C.E., Ponce, G., Vega, L.: Oscillatory integrals and regularity of dispersive equations. Indiana Univ. Math. J. 40, 33-69 (1991)

16. Kenig, C.E., Ponce, G., Vega, L.: The initial value problem for a class of non-linear dispersive equations. Lecture Notes in Math. vol. 1450, 141-156 (1990)

17. Kenig, C.E., Ponce, G., Vega, L.: Well posedness of the initial value problem for the Korteweg-de Vries equation. J. Am. Math. Soc. 4, 323-347 (1991)

18. Marshall, B.: Mixed norm estimates for the Klein-Gordon equation. Proc. of Conf. on Harmonic analysis in honor of A. Zygmund, Wadsworth (1981), 638-649

19. Pecher, H.: $L^{p}$-Abschätzungen und klassische Lösungen für nichtlineare Wellengleichungen. I. Math. Z. 150, 159-183 (1976)

20. Pecher, H.: Non-linear small data scattering for the wave and Klein-Gordon equations. Math. Z. 185, 245-263 (1984)

21. Segal, I.E.: Space-time decay for solutions of wave equations. Adv. Math. 22, 304-311 (1976)

22. Strichartz, R.S.: Restriction of Fourier transform to quadratic surfaces and decay of solutions of wave equations. Duke Math. J. 44, 705-774 (1977)

23. Thomas, P.: A restriction theorem for the Fourier transform. Bull. AMS 81, 477-478 (1975)

24. Yajima, K.: Existence of solutions for Schrödinger evolution equations. Commun. Math. Phys. 110, $415-426$ (1987) 